IJMMS 30:12 (2002) 733-760

PII. S0161171202012243

http://ijmms.hindawi.com

(c) Hindawi Publishing Corp.

\title{
GEOMETRY WITHOUT TOPOLOGY AS A NEW CONCEPTION OF GEOMETRY
}

\author{
YURI A. RYLOV
}

Received 3 March 2001 and in revised form 4 July 2001

\begin{abstract}
A geometric conception is a method of a geometry construction. The Riemannian geometric conception and a new T-geometric one are considered. T-geometry is built only on the basis of information included in the metric (distance between two points). Such geometric concepts as dimension, manifold, metric tensor, curve are fundamental in the Riemannian conception of geometry, and they are derivative in the T-geometric one. T-geometry is the simplest geometric conception (essentially, only finite point sets are investigated) and simultaneously, it is the most general one. It is insensitive to the space continuity and has a new property: the nondegeneracy. Fitting the T-geometry metric with the metric tensor of Riemannian geometry, we can compare geometries, constructed on the basis of different conceptions. The comparison shows that along with similarity (the same system of geodesics, the same metric) there is a difference. There is an absolute parallelism in T-geometry, but it is absent in the Riemannian geometry. In T-geometry, any space region is isometrically embeddable in the space, whereas in Riemannian geometry only convex region is isometrically embeddable. T-geometric conception appears to be more consistent logically, than the Riemannian one.
\end{abstract}

2000 Mathematics Subject Classification: 51F99, 51K99.

1. Introduction. A conception of geometry is a method (a set of principles), used for the construction of geometry. We use the term "Euclidean geometry" as a collective concept with respect to the terms "proper Euclidean geometry" and "pseudoEuclidean geometry." In the first case, the eigenvalues of the metric tensor matrix have similar signs, in the second case, they have different signs. The same interrelation takes place between the terms "Riemannian geometry," "proper Riemannian geometry", and "pseudo-Riemannian geometry." The proper Euclidean geometry can be constructed on the basis of different geometric conceptions. For instance, we can use the Euclidean axiomatic conception (Euclidean axioms), or the Riemannian conception of geometry (dimension, manifold, metric tensor, curve). We can use the metric conception of geometry (topological space, metric, curve). In any case, we obtain the same proper Euclidean geometry. From the point of view of this geometry, it is of no importance which one of the possible geometric conceptions is used for the geometry construction. It means that the category of geometry conception is metageometric.

However, if we are going to generalize (to modify) the Euclidean geometry, it appears to be very important to determine which of the many possible geometric conceptions is to use for the generalization. The point is that generalization requires some modification of the original (fundamental) statements of geometry in the scope of the same geometric conception. As far as the fundamental statements differ from one 
geometric conception to another, we are forced to modify the different statements, which leads naturally to different results.

If we use the Euclidean geometric conception, which contains only axioms and no numerical characteristics, the only possible modification consists of changing some axioms by others. In this case, some new geometries appear, which hardly may be considered a generalization of the Euclidean geometry. They are rather its different modifications.

Some fundamental statements of the Riemannian geometric conception contain numerical characteristics, as far as we set the dimension $n$ and the metric tensor $g_{i k}$, $i, k=1,2, \ldots, n$, consisting of several functions of one point, that is, of one argument $x=\left\{x^{i}\right\}, i=1,2, \ldots, n$. Varying $n$ and $g_{i k}$, we obtain a class of Riemannian geometries, where each geometry is labeled by several functions of one point.

Recently, a new geometric conception of the Euclidean geometry construction was suggested, see $[9,11]$. The Euclidean geometry appears to be formulated only in terms of the metric $\rho$, setting distance between all pairs of points of the space. Such a geometric conception is the most general, in the sense that all information on geometry is concentrated on one function of two points. It is evident that one function of two points contains more information, than several functions of one point (it is supposed that the set of points is continual). At some choice of the point set $\Omega$, where the metric and the geometry are set, the $n$-dimensional Euclidean geometry appears. At another choice of the metric, another generalized geometry appears on the same set $\Omega$. This geometry will be referred to as tubular geometry, or briefly T-geometry. All things being equal, the set of all T-geometries appears to be more powerful, than the set of all Riemannian geometries. This conception will be referred to as T-geometric conception, although the term "metric conception of geometry" fits more.

The point is that this term has been occupied. By the metric (or generalized Riemannian) geometry $[2,4,15]$ is meant usually a geometry, constructed on the basis of the metric geometric conception, whose fundamental statements are topology and metric, that is, the metric is not set on an arbitrary set of points, but on the topological space, where, in particular, concepts of continuity and of a curve are defined.

What actually is happening is that the metric geometric conception contains an excess of fundamental statements. This excess appears as follows. Imagine that some conception $A$ of Euclidean geometry contains some set of independent fundamental statements $a$. Let $b$ be some set of corollaries of the fundamental statements $a$. Consider now the set $a \cup b$ as a set of fundamental statements of a geometric conception. It is another conception $A^{\prime}$ of the Euclidean geometry. Its fundamental statements $a \cup b$ are not independent. Now we can obtain the conception $A$, or some other geometric conception, depending on how the fundamental statements $a \cup b$ are used. By obtaining generalized geometries, we may not vary the fundamental statements independently. To avoid contradiction, we have to take into account mutual dependence of the fundamental statements.

If we know nothing about mutual dependence of fundamental statements $a \cup b$, the geometric conception may appear to be eclectic. We risk to obtain contradictions, or artificial constraints on the generalized geometries obtained. In the case of the metric conception of the proper Euclidean geometry, the statements of the properties 
of the topological space and those of the curve are corollaries of metrical statements. They may be removed completely from the set of fundamental statements of the conception.

However, there are problems connected with the fact that we have some preconceptions on what is the geometry in general. In particular, it is a common practice to consider that the concept of the curve is an attribute of any geometry, which is used for description of the real space (or space-time). It is incorrect, and manifests itself, in particular, in the imposition of some unjustified constraints (triangle inequality) on metric, which makes the situation difficult. These preconceptions have a metalogic character. They are connected with the association properties of the human thinking. Overcoming these preconceptions needs a serious analysis.

A cause for writing this paper is a situation, arising after the appearance and discussion of papers on T-geometry [9, 11], which mean essentially a construction of a new geometric conception. Such a situation took place in the second half of the nineteenth century, when the non-Euclidean geometries appeared, and the most part of the mathematical community considered sceptically the applications of the Riemannian (and non-Euclidean) geometry to the real space geometry. The appearance of Riemannian geometries meant the appearance of a new geometric conception. The reason of sceptical relation of the mathematical community to Riemannian geometry has not been analyzed up till now, though it was described in [5].

The appearance of a more general conception of geometry is important for applications of geometry. Geometry is a ground of the space-time model, and the appearance of a new more general geometric conception poses the question as to whether the microcosm space-time geometry has been chosen optimally. If the existing space-time geometry is not optimal, it must be revised. The space-time geometry revision is to be accompanied by a revision of basic statements of physics as a science founded on the space-time model. For instance, the appearance of Riemannian geometries and the realization of the fact that a new conception of geometry appears together with their appearance, has lead finally to a revision of the space-time conception and to the creation of the general relativity theory.

Until the appearance of T-geometries, there was only one uniform isotropic geometry suitable for the space-time description. It is the Minkowski geometry. An alternative to the Minkowski geometry to be anywhere reasonable did not exist. After realization of the fact that nondegenerate geometries (T-geometries) are as good as degenerate (Riemannian) geometries, a class of geometries suitable for the description of uniform isotropic space-time appears. This class includes the Minkowski geometry. The uniform isotropic geometries of this class are labeled by a function of one argument. Geometries of the class differ in a value and character of nondegeneracy. All geometries of this class except for the Minkowski geometry appear to be nondegenerate. In nondegenerate geometry, a motion of free particles appears to be initially stochastic, whereas in degenerate geometry it is initially deterministic. It is well known, that the motion of microparticles (electrons, protons, etc.) is stochastic. It seems incorrect to choose such a space-time model, where the microparticle motion is deterministic, and thereafter to introduce additional hypotheses (principles of quantum mechanics), providing stochasticity of microparticle motion. It is more reasonable 
to choose at once such a space-time geometry which provides the microparticle motion stochasticity. It is desirable to choose from the class of uniform nondegenerate geometries, precisely that geometry which agrees optimally with experimental data. If the complete agreement with experimental data appears to be impossible, we can add supplementary hypotheses, as it is made in quantum mechanics. In any case, the space-time geometry is to be chosen optimally. The choice of the Minkowski geometry as a space-time model for microcosm is not optimal certainly. A use of the Minkowski geometry as a space-time model for microcosm is explained by the absence of alternative (i.e., essentially by a use of the Riemannian conception of geometry).

Thus, after the appearance of a new conception of geometry and the appearance of an alternative to the Minkowski geometry, a revision of the space-time model is a logical necessity. This revision must be carried out independently of that whether the new version of the space-time model explains all quantum effects, or only part of them. In the last case, we should add some hypotheses explaining that part of experimental data, which is not explained by the revised space-time model. In any case, we should use the most suitable space-time geometry among all possible ones.

Note that this conclusion does not agree with the viewpoint of most of physicists, dealing with relativistic quantum theory. Many of them suppose that any revision of the existing space-time model is justified only in the case, it explains at least one of the experiments that cannot be explained by the existing theory. We agree with such a position, provided that the existing theory modification does not concern the principles of the quantum theory and space-time model. At the appearance of a more general conception of geometry, we are forced to choose an optimal geometry independently of whether the new model solves all problems, or only part of them. Another viewpoint: when anybody suggests either to solve all problems by means of a revision of the space-time geometry, or, if it appears to be impossible, to abandon from revision at all and to use certainly nonoptimal geometry, which seems to be too extremistic.

Now results of the application of nondegenerate geometry for the space-time description seem to be rather optimistic, because we succeeded to choose such a nondegenerate geometry containing the quantum constant $\hbar$ as a parameter, that statistical description of stochastic particle motion in this space-time coincides with the quantum description in terms of Schrödinger equation in the conventional space-time model [10, 12]. Further development of the conception will show whether explanation of relativistic quantum effects is possible.

In the present paper, a new geometric conception based on the concept of distance and only distance is considered. In general, the idea of the geometry construction on the basis of the distance is natural and not new. The geometric conception, where the distance (metric) is a basic concept, is natural to be referred to as metric conception of geometry. Usually the term "metric geometry" is used for a geometry constructed on the base of the metric space.

DEFINITION 1.1. The metric space $M=\{\rho, \Omega\}$ is the set $\Omega$ of points $P \in \Omega$, equipped by the metric $\rho$, setting on $\Omega \times \Omega$

$$
\begin{gathered}
\rho: \Omega \times \Omega \rightarrow D_{+} \subset \mathbb{R}, \quad D_{+}=[0, \infty), \\
\rho(P, P)=0, \quad \rho(P, Q)=\rho(Q, P), \quad \forall P, Q \in \Omega,
\end{gathered}
$$




$$
\begin{gathered}
\rho(P, Q)=0, \quad \text { iff } P=Q, \forall P, Q \in \Omega, \\
\rho(P, Q)+\rho(Q, R) \geq \rho(P, R), \quad \forall P, Q, R \in \Omega .
\end{gathered}
$$

There is a generalization of metric geometry, known as distance geometry [3], which differs from the metric geometry in absence of constraints (1.3). The main problem of the metric geometric conception is a construction of geometric objects, that is, different sets of points in the metric space. For instance, to construct such a geometric object as the shortest, we are forced to introduce the concept of a curve as a continuous mapping of a segment of real axis on the space

$$
L: I \rightarrow \Omega, \quad I=[0,1] \subset \mathbb{R}
$$

The shortest, passing through points $P$ and $Q$, is defined as a curve segment of the shortest length. On the one hand, the introduction of the concept of a curve means a rejection from the pure metric conception of geometry, as far as we are forced to introduce concepts that are not defined via metric. On the other hand, if the concept of a curve is not introduced, it is not clear how to build such geometric objects that are analogs of Euclidean straight and plane. Without the introduction of these objects, the metric geometry looks as a very poor (slightly informative) geometry. Such a geometry cannot be used as a model of the real space-time.

Essentially, the problem of constructing a pure metric conception of geometry is set as follows. Is it possible to construct on the basis of only metric such a geometry that was as informative as the Euclidean geometry? In other words, is it possible to construct the Euclidean geometry, setting in some way the metric on $\Omega \times \Omega$, where $\Omega$ is a properly chosen set of points? More concretely this problem is formulated as follows.

Let $\rho_{E}$ be the metric of $n$-dimensional proper Euclidean space on $\Omega \times \Omega$. Is it possible on the base of information contained in $\rho_{E}$ to reconstruct the Euclidean geometry, that is, to determine the dimension $n$, to introduce rectilinear coordinate system and metric tensor in it, to construct $k$-dimensional planes $k=1,2, \ldots, n$, and to test whether the reconstructed geometry is proper Euclidean? If yes, and the information contained in metric is sufficient for the construction of proper Euclidean geometry, the used prescriptions can be used for the construction of a geometry with other metric. As a result each metric $\rho$ corresponds to some metric geometry $T_{\rho}$, constructed on the base of the metric and only metric. Any such a geometry $T_{\rho}$ is not less informative than the proper Euclidean one in the sense that any geometric object in the proper Euclidean geometry corresponds to a geometric object in the metric geometry $T_{\rho}$ constructed according to the same prescriptions as those of the proper Euclidean geometry. This geometric object may appear to bear little resemblance to its Euclidean analog. Besides, due to the symmetry of the Euclidean space (presence of a motion group), different geometric objects in $T_{\rho}$ may have the same Euclidean analog. For instance, in the Euclidean geometry any two different points $P$ and $Q$, lying on the Euclidean straight $L$, determine this straight. In metric geometry $T_{\rho}$ analogs of the Euclidean straight $\mathscr{T}_{P Q}, \mathscr{T}_{P_{1} Q_{1}}, P_{1}, Q_{1} \in \mathscr{T}_{P Q}$, determined by different pairs $P, Q$ and $P_{1}, Q_{1}$, are different, in general, if the metric does not satisfy condition (1.3). 
There exists a positive solution of the stated problem, that is, the amount of information, contained in the metric, is sufficient for constructing the metric geometry which is not less informative, than the Euclidean one. The corresponding theorem has been proved [13].

Apparently, Menger [6] succeeded to approach most closely the positive solution of the mentioned problem, but he failed to solve it completely. The reason of his failure is some delusion, which may be qualified as "associative prejudice." An overcoming of this prejudice results a construction of a new geometric conception, where all information on geometry are contained in metric. The new conception generates a class of T-geometries, which may be considered a generalization of conventional metric geometry on the base of the metric space $M=\{\rho, \Omega\}$. Formally this generalization is approached at the expense of reducing the number of fundamental concepts that are necessary for the geometry construction, and at the expense of elimination of constraints (1.2) and (1.3) imposed on metric. Besides, instead of the metric $\rho$ we use the quantity $\sigma=(1 / 2) \rho^{2}$, known as world function [14]. The world function is supposed to be real. It means that the metric $\rho$ may be either nonnegative, or pure imaginary quantity. This extends the capacities of geometry. Now we can consider the Minkowski geometry as a special case of T-geometry and use this latter as a space-time geometry. The concept of the curve (1.4) is not used at the construction of geometry, that is, it is not a fundamental concept; although the geometry construction has been completed, nothing prevents from introducing the curve by means of the mapping (1.4).

But the curve $L$ appears to be not an attribute of geometry. It is some additional object external with respect to geometry. A corollary of this is an appearance of a new geometry property, which is referred to as nondegeneracy. Euclidean and Riemannian geometries have no nondegeneracy. They are degenerate geometries. Associative prejudice is a delusion appearing when properties of one object are attributed by mistake to another object. We illustrate by a simple example, which is perceived now as a grotesque. It is known that Ancient Egyptians believed that all rivers flow towards the North. This delusion seems now to be nonsense. But many years ago it had a weighty foundation. The Ancient Egyptians lived on a vast flat plane and knew only one river: the Nile, which flew exactly towards the North and had no tributaries on the Egyptian territory. The North direction was a preferred direction for Ancient Egyptians who observed motion of heavenly bodies regularly. It was the direction toward the fixed North star. They did not connect the direction of the river flow with the plane slope, as we do now. They connected the direction of the river flow with the preferred spatial direction towards the North. We are interested now in that kind of mistake made Ancient Egyptians, believing that all rivers flow towards the North, and how could they overcome their delusion.

Their delusion was not a logical mistake, because the logic has no relation to this mistake. The delusion was connected by an associative property of human thinking, when the property $A$ is attributed to the object $B$ on the basis that in all known cases the property $A$ accompanies the object $B$. Such an association may be correct or not. If it is erroneous, as in the given case, it is very difficult to discover the mistake. At any rate it is difficult to discover the mistake by means of logic, because such associations appear before the logical analysis, and the subsequent logical analysis is carried out 
on the basis of the existing associations. Imagine that in the course of a voyage, an Ancient Egyptian scientist arrived the Tigris, which is the nearest to Egypt river. He discovers a water stream which flows, first, not outright and, second, not towards the North. Does he discover his delusion? Most likely not. At any rate not at once. He starts to think that the water stream, flowing before him, is not a river. A ground for such a conclusion is his initial belief that "real" river is to flow, first, directly and, second, towards the North. Besides, the Nile was very important in the life of Ancient Egyptians, and they were often apt to idolize the Nile. The delusion about the direction of the river flow can be overcome only after that, when one has discovered sufficiently many different rivers, flowing towards different directions, and the proper analysis of this circumstance has been carried out.

Thus, to overcome the associating delusion, it is not sufficient to present another object $B$, which has not the property $A$, because we may doubt of whether the presented object is to be classified really as the object $B$. Another attendant circumstances are also possible.

If the established association between the object and its property is erroneous, we can say it is an associative delusion or an associative prejudice. The usual method of overcoming the associative prejudices is a consideration of wider set of phenomena, where the established association between the property $A$ and the object $B$ may appear to be violated, and the associative prejudice is discovered.

The associative prejudices are very stable. It is very difficult to overcome them, when they have been established, because they cannot be disproved logically. On the other hand, fixing incorrect correlations between objects of real world, the associative prejudices point out a wrong way for investigations.

Associative prejudices are known in the history of science. For instance, the known statement of the Ptolemaic doctrine that the Earth is placed in the centre of universe, and heaven bodies rotate around it, is an example of the associative prejudice. In this case, the property of being a centre of a planetary system is attributed to the Earth, whereas such a centre is the Sun. Overcoming this prejudice was long and difficult, because in contrast to the prejudice of Ancient Egyptians it can be disproved neither logically, nor experimentally.

Another example of associative prejudice is that in the nineteenth century the popular opinion that the Cartesian coordinate system is an attribute of geometry. This viewpoint appeared, when the analytic geometry was discovered, and the Cartesian coordinate system became to serve as a tool in description of geometric objects of Euclidean geometry. Using analytic description of Euclidean geometry, many mathematicians of the nineteenth century applied Cartesian coordinates almost always and were inclined to believe that the Cartesian coordinates are an attribute of any geometry at all. On the other hand, nonuniform (Riemannian) geometry cannot be constructed in the Cartesian coordinate system. Any attempt to write the Riemannian geometry metric tensor in a Cartesian coordinates turns nonuniform (Riemannian) geometry to uniform (i.e., Euclidean) geometry. In other words, the Cartesian coordinate system discriminates any nonuniform geometry. It is known [5] that the mathematicians of nineteenth century were biased against the consideration of the Riemannian geometry as a really existing geometry. It seems that this skepticism in the relation of 
Riemannian geometry is connected with the associative prejudice, when the Cartesian coordinate system is considered to be an attribute of any geometry. As the coordinate system appears to be a way of the geometry description but its attribute, the scepticism disappears.

Now the viewpoint that the concept of the curve (1.4) is a fundamental concept (i.e., it is applied to the construction of any geometry) holds much favor. This viewpoint is based on the circumstance that the curve is used in the construction of all known (Riemannian and metric) geometries. Such a viewpoint is an associative prejudice (of the kind of the statement of Ancient Egyptians that all rivers flow towards the North). To prove this, it is sufficient to construct a sufficiently informative geometry without using the curve concept (1.4). Such a geometry (T-geometry) has been constructed [9]. Constructing the new conception of geometry, its author did not think that he did not use the curve concept and overcame some prejudice. The point is that the metric $\rho(x, y)$, considered to be a function of two variable points $x$ and $y$, contains much more information than the metric tensor $g_{i k}(x)$, which is several functions of one variable point $x$. The author believed that the information contained in the metric is sufficient for constructing geometry, and he wanted to construct a geometry on the base of only this information. It is possible, provided that the curve concept is ignored. He did not suspect that he overcame the associative preconception on fundamental role of the curve and, hence, created a new conception of geometry. All this became clear well later at the realization and discussion of the obtained results.

In the second section, the T-geometric technique is described, and we show that the Euclidean geometry can be formulated in terms of only metric. The method of the geometric objects construction in T-geometry is described in the third section. The fourth section is devoted to the convexity problem. In the fifth and sixth sections, we compare solutions of the parallelism problem in Riemannian and tubular geometries.

2. $\sigma$-space and T-geometry. T-geometry is constructed on $\sigma$-space $V=\{\sigma, \Omega\}$, which is obtained from the metric space after removal of constraints (1.2) and (1.3) and the introduction of the world function $\sigma$

$$
\sigma(P, Q) \equiv \frac{1}{2} \rho^{2}(P, Q), \quad \forall P, Q \in \Omega,
$$

instead of the metric $\rho$.

Definition 2.1. The $\sigma$-space $V=\{\sigma, \Omega\}$ is a nonempty set $\Omega$ of points $P$ with given on $\Omega \times \Omega$ real function $\sigma$

$$
\sigma: \Omega \times \Omega \longrightarrow \mathbb{R}, \quad \sigma(P, P)=0, \quad \sigma(P, Q)=\sigma(Q, P) \quad \forall P, Q \in \Omega .
$$

The function $\sigma$ is known as the world function [14], or $\sigma$-function. The metric $\rho$ may be introduced in the $\sigma$-space by means of the relation (2.1). If $\sigma$ is positive, the metric $\rho$ is also positive, but if $\sigma$ is negative, the metric is imaginary.

Definition 2.2. The nonempty point set $\Omega^{\prime} \subset \Omega$ of $\sigma$-space $V=\{\sigma, \Omega\}$ with the world function $\sigma^{\prime}=\left.\sigma\right|_{\Omega^{\prime} \times \Omega^{\prime}}$, which is a contraction $\sigma$ on $\Omega^{\prime} \times \Omega^{\prime}$, is called $\sigma$-subspace $V^{\prime}=\left\{\sigma^{\prime}, \Omega^{\prime}\right\}$ of $\sigma$-space $V=\{\sigma, \Omega\}$. 
Further, the world function $\sigma^{\prime}=\left.\sigma\right|_{\Omega^{\prime} \times \Omega^{\prime}}$, which is a contraction of $\sigma$ will be denoted $\sigma$. Any $\sigma$-subspace of the $\sigma$-space is a $\sigma$-space.

DeFINITION 2.3. The $\sigma$-space $V=\{\sigma, \Omega\}$ is called isometrically embeddable in the $\sigma$-space $V^{\prime}=\left\{\sigma^{\prime}, \Omega^{\prime}\right\}$, if there exists such a monomorphism $f: \Omega \rightarrow \Omega^{\prime}$, that $\sigma(P, Q)=$ $\sigma^{\prime}(f(P), f(Q))$, for all $P$, for all $Q \in \Omega, f(P), f(Q) \in \Omega^{\prime}$.

Any $\sigma$-subspace $V^{\prime}$ of the $\sigma$-space $V=\{\sigma, \Omega\}$ is isometrically embeddable in it.

DefinItion 2.4. Two $\sigma$-spaces $V=\{\sigma, \Omega\}$ and $V^{\prime}=\left\{\sigma^{\prime}, \Omega^{\prime}\right\}$ are called to be isometric (equivalent), if $V$ is isometrically embeddable in $V^{\prime}$, and $V^{\prime}$ is isometrically embeddable in $V$.

Definition 2.5. The $\sigma$-space $M=\{\rho, \Omega\}$ is called a finite $\sigma$-space, if the set $\Omega$ contains a finite number of points.

Definition 2.6. The $\sigma$-subspace $M_{n}\left(\mathscr{P}^{n}\right)=\left\{\sigma, \mathscr{P}^{n}\right\}$ of the $\sigma$-space $V=\{\sigma, \Omega\}$, consisting of $n+1$ points $\mathscr{P}^{n}=\left\{P_{0}, P_{1}, \ldots, P_{n}\right\}$ is called the $n$th order $\sigma$-subspace.

The T-geometry is a set of all propositions on properties of $\sigma$-subspaces of $\sigma$-space $V=\{\sigma, \Omega\}$. Presentation of T-geometry is produced on the language, containing only references to $\sigma$-function and constituents of $\sigma$-space, that is, to its $\sigma$-subspaces.

DEFinition 2.7. A description is called $\sigma$-immanent, if it does not contain any reference to objects or concepts other than finite subspaces of the metric space and its world function (metric).

The description $\sigma$-immanence provides independence of the description on the method of description. In this sense, the $\sigma$-immanence of a description in T-geometry recalls the concept of covariance in Riemannian geometry. The covariance of some relation in Riemannian geometry means that the considered relation is valid in all coordinate systems and, hence, describes only the properties of the Riemannian geometry in itself. The covariant description provides a cutting-off from the coordinate system properties, considering the relation in all coordinate systems at once. The $\sigma$ immanence provides a truncation from the methods of description by the absence of a reference to objects, which do not relate to geometry itself (coordinate system, concept of curve, dimension).

The basic elements of T-geometry are the finite $\sigma$-subspaces $M_{n}\left(\mathscr{P}^{n}\right)$, that is, the finite sets

$$
\mathscr{P}^{n}=\left\{P_{0}, P_{1}, \ldots, P_{n}\right\} \subset \Omega
$$

The main characteristic of the finite $\sigma$-subspace $M_{n}\left(\mathscr{P}^{n}\right)$ is its length $\left|M\left(\mathscr{P}^{n}\right)\right|$.

Definition 2.8. The squared length $\left|M\left(\mathscr{P}^{n}\right)\right|^{2}$ of the $n$th order $\sigma$-subspace $M\left(\mathscr{P}^{n}\right) \subset \Omega$ of the $\sigma$-space $V=\{\sigma, \Omega\}$ is the real number.

$$
\left|M\left(\mathscr{P}^{n}\right)\right|^{2}=\left(n ! S_{n}\left(\mathscr{P}^{n}\right)\right)^{2}=F_{n}\left(\mathscr{P}^{n}\right),
$$


where $S_{n}\left(\mathscr{P}^{n}\right)$ is the volume of the $(n+1)$-edron, whose vertices are placed at points $\mathscr{P}^{n} \equiv\left\{P_{0}, P_{1}, \ldots, P_{n}\right\} \subset \Omega$, defined by means of relations

$$
\begin{gathered}
F_{n}: \Omega^{n+1} \longrightarrow \mathbb{R}, \quad \Omega^{n+1}=\bigotimes_{k=1}^{n+1} \Omega, \quad n=1,2, \ldots, \\
F_{n}\left(\mathscr{P}^{n}\right)=\operatorname{det}\left\|\left(\mathbf{P}_{0} \mathbf{P}_{i} \cdot \mathbf{P}_{0} \mathbf{P}_{k}\right)\right\|, \quad P_{0}, P_{i}, P_{k} \in \Omega, i, k=1,2, \ldots, n, \\
\left(\mathbf{P}_{0} \mathbf{P}_{i} \cdot \mathbf{P}_{0} \mathbf{P}_{k}\right) \equiv \Gamma\left(P_{0}, P_{i}, P_{k}\right) \equiv \sigma\left(P_{0}, P_{i}\right)+\sigma\left(P_{0}, P_{k}\right)-\sigma\left(P_{i}, P_{k}\right), \quad i, k=1,2, \ldots, n,
\end{gathered}
$$

where the function $\sigma$ is defined via the metric $\rho$ by the relation (2.1) and $\mathscr{P}^{n}$ denotes $n+1$ points (2.3).

The meaning of the written relations is as follows. In the special case, when the $\sigma$-space is the Euclidean space and its $\sigma$-function coincides with the $\sigma$-function of Euclidean space, any two points $P_{0}, P_{1}$ determine the vector $\mathbf{P}_{0} \mathbf{P}_{1}$, and the relation (2.7) is a $\sigma$-immanent expression for the scalar product $\left(\mathbf{P}_{0} \mathbf{P}_{i} \cdot \mathbf{P}_{0} \mathbf{P}_{k}\right)$ of two vectors. Then the relation (2.6) is the Gram's determinant for $n$ vectors $\mathbf{P}_{0} \mathbf{P}_{i}, i=1,2, \ldots, n$, and $S_{n}\left(\mathscr{P}^{n}\right)$ is the Euclidean volume of the $(n+1)$-edron with vertices at the points $\mathscr{P}^{n}$.

The idea of constructing the T-geometry is very simple. All relations of proper Euclidean geometry are written in the $\sigma$-immanent form and declared to be valid for any $\sigma$-function. This results that any relation of proper Euclidean geometry corresponds to some relation of T-geometry. It is important that in the relations declared to be of T-geometry, only the properties (2.1) were used. The special properties of the Euclidean $\sigma$-function are not to be taken into account. The metric part of these relations was formulated and proved by Menger [6]. We present this result in our designations in the form of theorem

THEOREM 2.9. The $\sigma$-space $V=\{\sigma, \Omega\}$ is isometrically embeddable in n-dimensional proper Euclidean space $E_{n}$, if and only if any $(n+2)$ th order $\sigma$-subspace $M\left(\Im^{n+2}\right) \subset \Omega$ is isometrically embeddable in $E_{n}$.

Unfortunately, the formulation of this theorem is not $\sigma$-immanent, as it contains a reference to $n$-dimensional Euclidean space $E_{n}$ which is not defined $\sigma$-immanently. A more constructive version of the $\sigma$-space Euclideaness conditions is formulated in the form

(I)

$$
\exists \mathscr{P}^{n} \subset \Omega, F_{n}\left(\mathscr{P}^{n}\right) \neq 0, \quad F_{n+1}\left(\Omega^{n+2}\right)=0,
$$

(II)

$$
\sigma(P, Q)=\frac{1}{2} \sum_{i, k=1}^{n} g^{i k}\left(\mathscr{P}^{n}\right)\left[x_{i}(P)-x_{i}(Q)\right]\left[x_{k}(P)-x_{k}(Q)\right], \quad \forall P, Q \in \Omega,
$$

where the quantities $x_{i}(P), x_{i}(Q)$ are defined by the relations

$$
x_{i}(P)=\left(\mathbf{P}_{0} \mathbf{P}_{i} \cdot \mathbf{P}_{0} \mathbf{P}\right), \quad x_{i}(Q)=\left(\mathbf{P}_{0} \mathbf{P}_{i} \cdot \mathbf{P}_{0} \mathbf{Q}\right), \quad i=1,2, \ldots, n .
$$


The contravariant components $g^{i k}\left(\mathscr{P}^{n}\right),(i, k=1,2, \ldots, n)$ of the metric tensor are defined by its covariant components $g_{i k}\left(\mathscr{P}^{n}\right),(i, k=1,2, \ldots, n)$ by means of the relations

$$
\sum_{k=1}^{n} g_{i k}\left(\mathscr{P}^{n}\right) g^{k l}\left(\mathscr{P}^{n}\right)=\delta_{i}^{l}, \quad i, l=1,2, \ldots, n,
$$

where

$$
g_{i k}\left(\mathscr{P}^{n}\right)=\Gamma\left(P_{0}, P_{i}, P_{k}\right), \quad i, k=1,2, \ldots, n .
$$

(III) The relations

$$
\Gamma\left(P_{0}, P_{i}, P\right)=x_{i}, \quad x_{i} \in \mathbb{R}, i=1,2, \ldots, n,
$$

considered to be equations for the determination of $P \in \Omega$, have always one and only one solution.

(IIIa) Relations (2.13), considered to be equations for determination of $P \in \Omega$, have always no more than one solution.

REMARK 2.10. Condition (2.8) is a corollary of condition (2.9). It is formulated in the form of a special condition, in order that a determination of the dimension being separated from the determination of coordinate system.

Condition (I) determines the space dimension. Condition (II) describes $\sigma$-immanently the scalar product properties of the proper Euclidean space. Setting $n+1$ points $\mathscr{P}^{n}$ satisfying condition (I), we determine $n$-dimensional basis of vectors in Euclidean space. Relations (2.11) and (2.12) determine covariant and contravariant components of the metric tensor, and relations (2.10) determine covariant coordinates of points $P$ and $Q$ on this basis. Relation (2.9) determines the expression for $\sigma$-function for two arbitrary points in terms of the coordinates of these points. Finally, condition (III) describes the continuity of the set $\Omega$ and a possibility to construct a manifold on it. Necessity of conditions (I), (II), and (III) for Euclideaness of the $\sigma$-space is evident. We can prove their sufficiency [13]. The connection of conditions (I), (II), and (III) with the Euclideaness of the $\sigma$-space can be formulated in the form of a theorem.

THEOREM 2.11. The $\sigma$-space $V=\{\sigma, \Omega\}$ is the $n$-dimensional Euclidean space, if and only if the $\sigma$-immanent conditions (I), (II), and (III) are fulfilled.

REMARK 2.12. If the $\sigma$-space is proper Euclidean, the eigenvalues of the matrix $g_{i k}\left(\mathscr{P}^{n}\right), i, k=1,2, \ldots, n$ must have the same sign, otherwise it is pseudo-Euclidean.

The theorem states that it is sufficient to know the metric (world function) to construct Euclidean geometry. The information, contained in the concepts of topological spaces and curves, used in metric geometry, appears to be excess.

The proof of this theorem can be found in [13]. A similar theorem for another (but close) necessary and sufficient conditions have been proved in [9]. Here, we show only constructive character of conditions (I), (II), and (III) for proper Euclidean space. It means that starting from an abstract $\sigma$-space satisfying conditions (I), (II), and (III), we can determine dimension $n$ and construct a rectilinear coordinate system with 
conventional description of the proper Euclidean space in it. We construct sequentially straight two-dimensional plane and so forth, up to the $n$-dimensional plane coincide with the set $\Omega$. To construct all these objects, we need to develop the technique of T-geometry.

DEFINITION 2.13. The finite $\sigma$-space $M_{n}\left(\mathscr{P}^{n}\right)=\left\{\sigma, \mathscr{P}^{n}\right\}$ is called oriented $\overrightarrow{M_{n}\left(\mathscr{P}^{n}\right)}$, if the order of its points $\mathscr{P}^{n}=\left\{P_{0}, P_{1}, \ldots, P_{n}\right\}$ is fixed.

DEFINITION 2.14. The $n$th order multivector $m_{n}$ is the mapping

$$
m_{n}: I_{n} \longrightarrow \Omega, \quad I_{n} \equiv\{0,1, \ldots, n\} .
$$

The set $I_{n}$ has a natural ordering, which generates an ordering of images $m_{n}(k) \in \Omega$ of points $k \in I_{n}$. The ordered list of images of points in $I_{n}$ has a one-to-one connection with the multivector and may be used as the multivector descriptor. Different versions of the point list will be used for writing the $n$th order multivector descriptor,

$$
\overrightarrow{P_{0} P_{1} \cdots P_{n}} \equiv \mathbf{P}_{0} \mathbf{P}_{1} \cdots \mathbf{P}_{n} \equiv \overrightarrow{\mathscr{P}^{n}}
$$

The originals of the points $P_{k}$ in $I_{n}$ are determined by the order of the point $P_{k}$ in the list of descriptor. Index of the point $P_{k}$ has nothing to do with the original of $P_{k}$. Further, we will use the descriptor, $\overrightarrow{P_{0} P_{1} \cdots P_{n}}$ of the multivector instead of the multivector. In this sense, the $n$th order multivector $\overrightarrow{P_{0} P_{1} \cdots P_{n}}$ in the $\sigma$-space $V=\{\sigma, \Omega\}$ may be defined as the ordered set $\left\{P_{l}\right\}, l=0,1, \ldots, n$ of $n+1$ points $P_{0}, P_{1}, \ldots, P_{n}$, belonging to the $\sigma$-space $V$. The point $P_{0}$ is the origin of the multivector $\overrightarrow{P_{0} P_{1} \cdots P_{n}}$. Suppose that $m_{n}\left(I_{n}\right)$ of the set $I_{n}$ contains $k$ points $(k \leq n+1)$. The set of all $n$th order multivectors $m_{n}$ constitutes the set $\Omega^{n+1}=\bigotimes_{k=1}^{n+1} \Omega$, and any multivector $\overrightarrow{\mathscr{P}^{n}} \in \Omega^{n+1}$.

DEFINITION 2.15. The scalar $\sigma$-product $\left(\overrightarrow{\mathscr{P}^{n}} \cdot \overrightarrow{2^{n}}\right)$ of two $n$th order multivectors $\overrightarrow{\mathscr{P}^{n}}$ and $\overrightarrow{2^{n}}$ is the real number

$$
\begin{aligned}
&\left(\overrightarrow{\mathscr{P}^{n}} \cdot \overrightarrow{\mathscr{Q}^{n}}\right)=\operatorname{det}\left\|\left(\mathbf{P}_{0} \mathbf{P}_{i} \cdot \mathbf{Q}_{0} \mathbf{Q}_{k}\right)\right\|, \quad i, k=1,2, \ldots, n, \overrightarrow{\mathscr{P}^{n}}, \overrightarrow{\mathscr{Q}^{n}} \in \Omega^{n+1}, \\
&\left(\mathbf{P}_{0} \mathbf{P}_{i} \cdot \mathbf{Q}_{0} \mathbf{Q}_{k}\right) \equiv \sigma\left(P_{0}, Q_{i}\right)+\sigma\left(Q_{0}, P_{k}\right)-\sigma\left(P_{0}, Q_{0}\right)-\sigma\left(P_{i}, Q_{k}\right), \\
& P_{0}, P_{i}, Q_{0}, Q_{k} \in \Omega .
\end{aligned}
$$

DEFINITION 2.16. The length $\left|\overrightarrow{\mathscr{P}^{n}}\right|$ of the $n$th order multivector $\overrightarrow{\mathscr{P}^{n}}$ is the number

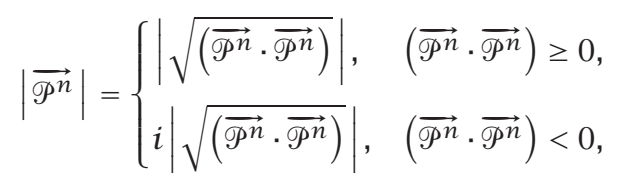

where $\overrightarrow{\mathscr{P}^{n}} \in \Omega^{n+1}$.

In the case when the multivector does not contain similar points, it coincides with the oriented finite $\sigma$-subspace, and it is a constituent of the $\sigma$-space. In the case when at least two points of the multivector coincide, the multivector length vanishes and the multivector is considered to be null multivector. The null multivector is not a finite 
$\sigma$-subspace, but a use of null multivectors assists in creating a more simple technique. Manipulation with numbers written in Arabic numerals (where zero is present) is simpler than the same manipulation with numbers written in Roman numerals (where zero is absent). Something like that takes place in the case of multivectors. Essentially, the multivectors are basic objects of T-geometry. As to continual geometric objects, which are the analogs of planes, spheres, ellipsoids, and so forth, they are constructed by means of skeleton-envelope method (see next section) with multivectors, or finite $\sigma$-subspaces used as skeletons. As a consequence, the T-geometry is presented $\sigma$-immanently, that is, without reference to objects, external with respect to the $\sigma$-space.

DEFINITION 2.17. Two $n$th order multivectors $\overrightarrow{\mathscr{P}^{n}} \overrightarrow{2^{n}}$ are collinear $\overrightarrow{\mathscr{P}^{n}} \| \overrightarrow{2^{n}}$, if

$$
\left(\overrightarrow{\mathscr{P}^{n}} \cdot \overrightarrow{2^{n}}\right)^{2}=\left|\overrightarrow{\mathscr{P}^{n}}\right|^{2} \cdot\left|\overrightarrow{2^{n}}\right|^{2} \text {. }
$$

DEFINITION 2.18. Two collinear $n$th order multivectors $\overrightarrow{\mathscr{P}^{n}}$ and $\overrightarrow{2^{n}}$ are similarly oriented $\overrightarrow{\mathscr{P}^{n}} \uparrow \overrightarrow{2^{n}}$ (parallel), if

$$
\left(\overrightarrow{\mathscr{P}^{n}} \cdot \overrightarrow{\mathscr{2}^{n}}\right)=\left|\overrightarrow{\mathscr{P}^{n}}\right| \cdot\left|\overrightarrow{\mathscr{2}^{n}}\right| .
$$

They have opposite orientation $\overrightarrow{\mathscr{P}^{n}} \uparrow \downarrow \overrightarrow{2^{n}}$ (antiparallel), if

$$
\left(\overrightarrow{\mathscr{P}^{n}} \cdot \overrightarrow{2^{n}}\right)=-\left|\overrightarrow{\mathscr{P}^{n}}\right| \cdot\left|\overrightarrow{2^{n}}\right| \text {. }
$$

The vector $\mathbf{P}_{0} \mathbf{P}_{1}=\overrightarrow{\mathscr{P P}^{1}}$ is the first order multivector.

DEFINITION 2.19. The $n$th order $\sigma$-subspace $M\left(\mathscr{P}^{n}\right)$ of nonzero length $\left|M\left(\mathscr{P}^{n}\right)\right|^{2}=$ $F_{n}\left(\mathscr{P}^{n}\right) \neq 0$ determines the set of points $\mathscr{T}\left(\mathscr{P}^{n}\right)$ called the $n$th order tube by means of the relation

$$
\mathscr{T}\left(\mathscr{P}^{n}\right) \equiv \mathscr{T}_{\mathscr{P} n}=\left\{P_{n+1} \mid F_{n+1}\left(\mathscr{P}^{n+1}\right)=0\right\}, \quad P_{i} \in \Omega, i=0,1 \ldots, n+1,
$$

where the function $F_{n}$ is defined by the relations (2.5), (2.6), and (2.7).

In arbitrary T-geometry, the $n$th order tube is an analog of the $n$-dimensional properly Euclidean plane.

Definition 2.20. The section $\mathscr{S}_{n ; P}$ of the tube $\mathscr{T}\left(\mathscr{P}^{n}\right)$ at the point $P \in \mathscr{T}\left(\mathscr{P}^{n}\right)$ is the set $\mathscr{T}_{n ; P}\left(\mathscr{T}\left(\mathscr{P}^{n}\right)\right)$ of points belonging to the tube $\mathscr{T}\left(\mathscr{P}^{n}\right)$

$$
\mathscr{S}_{n ; P}\left(\mathscr{T}\left(\mathscr{P}^{n}\right)\right)=\left\{P^{\prime} \mid \bigwedge_{l=0}^{l=n} \sigma\left(P_{l}, P^{\prime}\right)=\sigma\left(P_{l}, P\right)\right\}, \quad P \in \mathscr{T}\left(\mathscr{P}^{n}\right), P^{\prime} \in \Omega .
$$

Note that $\mathscr{S}_{n ; P}\left(\mathscr{T}\left(\mathscr{P}^{n}\right)\right) \subset \mathscr{T}\left(\mathscr{P}^{n}\right)$, because $P \in \mathscr{T}\left(\mathscr{P}^{n}\right)$. Indeed, whether the point $P$ belongs to $\mathscr{T}\left(\mathscr{P}^{n}\right)$ depends only on values of $n+1$ quantities $\sigma\left(P_{l}, P\right), l=0,1, \ldots, n$. In accordance with (2.23), these quantities are the same for both points $P$ and $P^{\prime}$. Hence, any running point $P^{\prime} \in \mathscr{T}\left(\mathscr{P}^{n}\right)$, if $P \in \mathscr{T}\left(\mathscr{P}^{n}\right)$.

In the proper Euclidean space, the $n$th order tube is an $n$-dimensional plane containing the points $\mathscr{P}^{n}$, and its section $\mathscr{S}_{n ; P}\left(\mathscr{T}\left(\mathscr{P}^{n}\right)\right)$ at the point $P$ consists of one point $P$. 
Now we can construct the proper Euclidean space and rectilinear coordinate system in it on the basis of only the $\sigma$-function. It is known that the $\sigma$-space $V=\{\sigma, \Omega\}$ is the proper Euclidean space, but its dimension is not known. To determine the dimension $n$, take two different points $P_{0}, P_{1} \in \Omega, F_{1}\left(\mathscr{P}^{1}\right)=2 \sigma\left(P_{0}, P_{1}\right) \neq 0$.

(1) Construct the first order tube $\mathscr{T}\left(\mathscr{P}^{1}\right)$. If $\mathscr{T}\left(\mathscr{P}^{1}\right)=\Omega$, then the dimension of the $\sigma$-space $V$ is $n=1$. If $\Omega \backslash \mathscr{T}\left(\mathscr{P}^{1}\right) \neq \varnothing$, there exists $P_{2} \in \Omega, P_{2} \notin \mathscr{T}\left(\mathscr{P}^{1}\right)$, and hence, $F_{2}\left(\mathscr{P}^{2}\right) \neq 0$.

(2) Construct the second order tube $\mathscr{T}\left(\mathscr{P}^{2}\right)$. If $\mathscr{T}\left(\mathscr{P}^{2}\right)=\Omega$ then $n=2$, otherwise there exists $P_{3} \in \Omega, P_{3} \notin \mathscr{T}\left(\mathscr{P}^{2}\right)$, and hence, $F_{3}\left(\mathscr{P}^{3}\right) \neq 0$.

(3) Construct the third order tube $\mathscr{T}\left(\mathscr{P}^{3}\right)$. If $\mathscr{T}\left(\mathscr{P}^{3}\right)=\Omega$ then $n=3$, otherwise there exists $P_{4} \in \Omega, P_{4} \notin \mathscr{T}\left(\mathscr{P}^{3}\right)$, and hence, $F_{4}\left(\mathscr{P}^{4}\right) \neq 0$.

(4) And so forth.

Continuing this process, we determine such $n+1$ points $\mathscr{P}^{n}$ that the condition $\mathscr{T}\left(\mathscr{P}^{n}\right)=\Omega$ and, hence, conditions (2.8) are fulfilled. Then by means of the relation

$$
x_{i}(P)=\Gamma\left(P_{0}, P_{i}, P\right), \quad i=1,2, \ldots n,
$$

we attribute the covariant coordinates $x(P)=\left\{x_{i}(P)\right\}, i=1,2, \ldots, n$ to all $P \in \Omega$. Let $x=x(P) \in \mathbb{R}^{n}$ and $x^{\prime}=x\left(P^{\prime}\right) \in \mathbb{R}^{n}$. Substituting $\Gamma\left(P_{0}, P_{i}, P\right)=x$ and $\Gamma\left(P_{0}, P_{i}, P^{\prime}\right)=x_{i}^{\prime}$ in (2.9), we obtain the conventional expression for the world function of the Euclidean space in the rectilinear coordinate system

$$
\sigma\left(P, P^{\prime}\right)=\sigma_{E}\left(x, x^{\prime}\right)=\frac{1}{2} \sum_{i, k=1}^{n} g^{i k}\left(\mathscr{P}^{n}\right)\left(x_{i}-x_{i}^{\prime}\right)\left(x_{k}-x_{k}^{\prime}\right),
$$

where $g^{i k}\left(\mathscr{P}^{n}\right)$, defined by the relations (2.12) and (2.11), is the contravariant metric tensor in this coordinate system.

Condition (III) of Theorem 2.11 states that the mapping

$$
x: \Omega \longrightarrow \mathbb{R}^{n}
$$

described by the relation (2.24), is a bijection, that is, for all $y \in \mathbb{R}^{n}$ there exists one and only one point $Q \in \Omega$, such that $y=x(Q)$.

Thus, on the base of the world function given on the abstract set $\Omega \times \Omega$, we can determine the dimension $n$ of the Euclidean space, construct rectilinear coordinate system with the metric tensor $g_{i k}\left(\mathscr{P}^{n}\right)=\Gamma\left(P_{0}, P_{i}, P_{k}\right), i, k=1,2, \ldots, n$, and describe all geometrical objects which are determined in terms of coordinates. The Euclidean space and Euclidean geometry are described in terms and only in terms of the world function (metric).

Conditions (I), (II), and (III), formulated in the $\sigma$-immanent form, admit to construct the proper Euclidean space, using only information contained in the world function. The $\sigma$-immanence of the formulation admits us to state that information contained in the world function, is sufficient for the construction of any T-geometry. Substitution of condition (III) by the condition (IIIa) leads to a reduction of constraints. At the fulfillment of conditions (I), (II), and (IIIa) the $\sigma$-space appears to be isometrically embeddable in $n$-dimensional Euclidean space. It may be piecewise continuous, or 
even discrete. Such a $\sigma$-space can be obtained, removing arbitrary number of points from $n$-dimensional Euclidean space.

\section{Skeleton-envelope method of geometric objects construction}

DeFINITION 3.1. A geometric object $\mathcal{O}$ is some $\sigma$-subspace of the $\sigma$-space.

In T-geometry, a geometric object $\mathcal{O}$ is described by means of the skeleton-envelope method. It means that any geometric object $\mathcal{O}$ is considered to be a set of intersections and joins of elementary geometric objects (EGO).

Definition 3.2. An elementary geometric object $\mathscr{E} \subset \Omega$ is a set of zeros of the envelope function

$$
f_{\mathscr{P} n}: \Omega \longrightarrow \mathbb{R}, \quad \mathscr{P}^{n} \equiv\left\{P_{0}, P_{1}, \ldots, P_{n}\right\} \subset \Omega,
$$

that is,

$$
\mathscr{E}=\mathscr{E}_{f}\left(\mathscr{P}^{n}\right)=\left\{R \mid f_{\mathscr{P} n}(R)=0\right\} .
$$

The finite set $\mathscr{P}^{n} \subset \Omega$ of parameters of the envelope function $f_{\mathscr{P}}^{n}$ is a skeleton of EGO $\mathscr{E} \subset \Omega$. The set $\mathscr{E} \subset \Omega$ of points forming EGO is called the envelope of its skeleton $\mathscr{P}^{n}$. For continuous T-geometry the envelope $\mathscr{E}$ is usually a continual set of points. The envelope function $f_{\text {ø } n}$, determining EGO is a function of the running point $R \in \Omega$ and of parameters $\mathscr{P}^{n} \subset \Omega$. The envelope function $f_{\mathscr{\rho} n}$ is supposed to be an algebraic function of $s$ arguments $w=\left\{w_{1}, w_{2}, \ldots, w_{s}\right\}, s=(n+2)(n+1) / 2$. Each of the arguments $w_{k}=\sigma\left(Q_{k}, L_{k}\right)$ is a $\sigma$-function of two arguments $Q_{k}, L_{k} \in\left\{R, \mathscr{P}^{n}\right\}$, either belonging to the skeleton $\mathscr{P}^{n}$, or coinciding with the running point $R$.

Consider examples of some simplest EGOs,

$$
\mathscr{S}\left(P_{0}, P_{1}\right)=\left\{R \mid f_{P_{0} P_{1}}(R)=0\right\}, \quad f_{P_{0} P_{1}}(R)=\sqrt{2 \sigma\left(P_{0}, P_{1}\right)}-\sqrt{2 \sigma\left(P_{0}, R\right)}
$$

is a sphere, passing through the point $P_{1}$ and having its center at the point $P_{0}$. The ellipsoid $\mathscr{E} \mathscr{L}$, passing through the point $P_{2}$ and having the focuses at the points $P_{0}, P_{1}$ $\left(P_{0} \neq P_{1}\right)$ is described by the relation

$$
\mathscr{E} \mathscr{L}\left(P_{0}, P_{1}, P_{2}\right)=\left\{R \mid f_{P_{0} P_{1} P_{2}}(R)=0\right\},
$$

where the envelope function $f_{P_{0} P_{1} P_{2}}(R)$ is defined by the equation,

$$
f_{P_{0} P_{1} P_{2}}(R)=\sqrt{2 \sigma\left(P_{0}, P_{2}\right)}+\sqrt{2 \sigma\left(P_{1}, P_{2}\right)}-\sqrt{2 \sigma\left(P_{0}, R\right)}-\sqrt{2 \sigma\left(P_{1}, R\right)} .
$$

If the focuses at $P_{0}, P_{1}$ coincide $\left(P_{0}=P_{1}\right)$, the ellipsoid $\mathscr{E} \mathscr{L}\left(P_{0}, P_{1}, P_{2}\right)$ degenerates into a sphere $\mathscr{S}\left(P_{0}, P_{2}\right)$. If the points $P_{1}, P_{2}$ coincide $\left(P_{1}=P_{2}\right)$, the ellipsoid $\mathscr{E} \mathscr{L}\left(P_{0}, P_{1}, P_{2}\right)$ degenerates into a tube segment $\mathscr{T}_{\left[P_{0} P_{1}\right]}$ between the points $P_{0}, P_{1}$ of the tube $\mathscr{T}_{P_{0} P_{1}}$,

$$
\begin{gathered}
\mathscr{T}_{\left[P_{0} P_{1}\right]}=\mathscr{E} \mathscr{L}\left(P_{0}, P_{1}, P_{1}\right)=\left\{R \mid f_{P_{0} P_{1} P_{1}}(R)=0\right\}, \\
f_{P_{0} P_{1} P_{1}}(R)=S_{2}\left(P_{0}, R, P_{1}\right) \equiv \sqrt{2 \sigma\left(P_{0}, P_{1}\right)}-\sqrt{2 \sigma\left(P_{0}, R\right)}-\sqrt{2 \sigma\left(P_{1}, R\right)} .
\end{gathered}
$$


In the proper Euclidean geometry, $\mathscr{T}_{\left[P_{0} P_{1}\right]}$ is simply a segment of the straight between the points $P_{0}, P_{1}$.

The most important and interesting EGOs arise, when values of the envelope function $f_{\mathscr{}} n(R)$ coincide with those of the function $F_{n+1}\left(\mathscr{P}^{n}, R\right)$, determined by relation (2.6) and proportional to the squared length of the finite $\sigma$-subspace, consisting of $n+2$ points $\mathscr{P}^{n}, R$. This object is called the $n$th order natural geometric object (NGO). It is defined by the relation (2.22). In the case of proper Euclidean geometry, it coincides with an $n$-dimensional plane.

Another function $f$ generates another envelope of elementary geometrical objects for the given skeleton $\mathscr{P}^{n}$. For instance, the set of two points $\left\{P_{0}, P_{1}\right\}$ forms a skeleton not only for the tube $\mathscr{T}_{P_{0} P_{1}}$, but also for the segment $\mathscr{T}_{\left[P_{0} P_{1}\right]}$ of the tube (straight) (3.6), and for the tube ray $\mathscr{T}_{\left[P_{0} P_{1}\right]}$, which is defined by the relation

$$
\mathscr{T}_{\left[P_{0} P_{1}\right]}=\left\{R \mid S_{2}\left(P_{0}, P_{1}, R\right)=0\right\},
$$

where the function $S_{2}$ is defined by relation (3.7).

\section{Interrelation between T-geometric and Riemannian conceptions of geometry}

Definition 4.1. The geometric conception is a totality of principles of the geometry construction.

Compare the Riemannian conception of geometry and that of T-geometry. The $n$ dimensional Riemannian geometry $R_{n}=\left\{\mathbf{g}, K, \mu_{n}\right\}$ is introduced on $n$-dimensional manifold $\mu_{n}$ in some coordinate system $K$ by setting the metric tensor $g_{i k}(x)$, $i, k=1,2, \ldots, n$. Thereafter, using definition (1.4) of the curve that always can be introduced on the manifold $\mathcal{M}_{n}$, we introduce the concept of geodesic $\mathscr{L}_{\left[x x^{\prime}\right]}$ as the shortest curve connecting points with coordinates $x$ and $x^{\prime}$. In the Riemannian space $R_{n}=\left\{\mathbf{g}, K, \mu_{n}\right\}$ we introduce the world function $\sigma_{R}\left(x, x^{\prime}\right)$ between points $x$ and $x^{\prime}$ defined by the relation

$$
\sigma_{R}\left(x, x^{\prime}\right)=\frac{1}{2}\left(\int_{\mathscr{L}_{\left[x x^{\prime}\right]}} \sqrt{g_{i k} d x^{i} d x^{k}}\right)^{2},
$$

where $\mathscr{L}_{\left[x x^{\prime}\right]}$ denotes a segment of geodesic connecting points $x$ and $x^{\prime}$.

T-geometry can be introduced on any set $\Omega$, including the manifold $\mu_{n}$. To set Tgeometry on $\mu_{n}$, it is insufficient to introduce the metric tensor $g_{i k}(x), i, k=1,2, \ldots, n$, because it determines only the first derivatives of the world function at coinciding points

$$
g_{i k}(x)=-\sigma_{i k^{\prime}}(x, x) \equiv-\left[\frac{\sigma\left(x, x^{\prime}\right)}{\partial x^{i} \partial x^{\prime k}}\right]_{x^{\prime}=x} .
$$

This is insufficient for the determination of the world function. For setting T-geometry in a way to be consistent with the Riemannian geometry, we should set $\sigma\left(x, x^{\prime}\right)=$ $\sigma_{R}\left(x, x^{\prime}\right)$, where $\sigma_{R}\left(x, x^{\prime}\right)$ is defined by relation (4.1). Now we can construct geometric objects by the method described above. The T-geometry, introduced in such a way, will be referred to as $\sigma$-Riemannian geometry, to distinguish the different conceptions (i.e., rules of construction) of geometry. 
Note that the world function, consistent with Riemannian geometry on the manifold, may be set as a solution of equations in partial derivatives. For instance, the world function can be defined as the solution of the differential equation [14]

$$
\sigma_{i} g^{i k}(x) \sigma_{k}=2 \sigma, \quad \sigma_{i} \equiv \frac{\partial \sigma}{\partial x^{i}}, \quad i=1,2, \ldots, n,
$$

satisfying conditions (2.2).

The basic geometric objects of Riemannian geometry, geodesic segments $\mathscr{L}_{\left[x x^{\prime}\right]}$, coincide with the first order NGOs in T-geometry, the tube segments $\mathscr{T}_{\left[x x^{\prime}\right]}$ defined by relations (3.6). Thus we can say upon this partial coincidence of the two geometric conceptions (Riemannian and $\sigma$-Riemannian ones). But such a coincidence is not complete. There are some difference that appears sometimes essential.

Consider the case where the manifold $\mu_{n}$ coincides with $\mathbb{R}^{n}$ and metric tensor $g_{i k}=$ const, $i, k=1,2, \ldots, n, g=\operatorname{det}\left\|g_{i k}\right\| \neq 0$ is the metric tensor of the proper Euclidean space. The world function is described by relation (2.25), and the proper Riemannian space $E_{n}=\left\{\mathbf{g}_{E}, K, \mathbb{R}^{n}\right\}$ is the proper Euclidean space. Here $\mathbf{g}_{E}$ denotes the metric tensor of the proper Euclidean space.

Now consider the proper Riemannian space $R_{n}=\left\{\mathbf{g}_{E}, K, D\right\}$, where $D \subset \mathbb{R}^{n}$ is some region of the proper Euclidean space $E_{n}=\left\{\mathbf{g}_{E}, K, \mathbb{R}^{n}\right\}$. If this region, $D$ is convex, that is, any segment $\mathscr{L}_{\left[x x^{\prime}\right]}$ of a straight passing through points $x, x^{\prime} \in D$ belongs to $D\left(\mathscr{L}_{\left[x x^{\prime}\right]} \subset D\right)$, the world function of the proper Riemannian space $R_{n}=\left\{\mathbf{g}_{E}, K, D\right\}$ has the form (2.25), and the proper Riemannian space $R_{n}=\left\{\mathbf{g}_{E}, K, D\right\}$ can be embedded isometrically to the proper Euclidean space $E_{n}=\left\{\mathbf{g}_{E}, K, \mathbb{R}^{n}\right\}$.

If the region $D$ is not convex, the system of geodesics in the region $R_{n}=\left\{\mathbf{g}_{E}, K, D\right\}$ is not a system of straights, and world function (4.1) is not described by relation (2.25). In this case, the region $D$ cannot be embedded isometrically in $E_{n}=\left\{\mathbf{g}_{E}, K, \mathbb{R}^{n}\right\}$, in general. It seems to be paradoxical that one (nonconvex) part of the proper Euclidean space cannot be embedded isometrically to it, whereas another (convex) part can.

The convexity problem appears to be rather complicated, and most of mathematicians prefer to go around this problem, dealing only with convex regions [1]. In Tgeometry there is no convexity problem. Indeed, according to Definition 2.2 a subset of points of $\sigma$-space is always embeddable isometrically in the $\sigma$-space. From the viewpoint of T-geometry, a removal of any region $R_{n}=\left\{\mathbf{g}_{E}, K, D\right\}$ from the proper Euclidean space $R_{n}=\left\{\mathbf{g}_{E}, K, \mathbb{R}^{n}\right\}$ cannot change shape of geodesics (first order NGOs). It leads only to holes in geodesics, making them discontinuous. The continuity is a property of the coordinate system, used in the proper Riemannian geometry as a main tool of description. Using continuous coordinate systems for description, we transfer constraints imposed on coordinate system to the geometry itself.

Insisting on continuity of geodesics, we overestimate the importance of continuity for geometry and attribute continuous geodesics (the first order NGOs) to any proper Riemannian geometry, whereas the continuity is a special property of the proper Euclidean geometry. From the viewpoint of T-geometry, the convexity problem is an artificial problem. The existence of the convexity problem in the Riemannian conception of geometry and its absence from the T-geometric conception means that the second conception of geometry is more perfect. 
5. Collinearity in Riemannian and $\sigma$-Riemannian geometry. We return to the Riemannian space $R_{n}=\{\mathbf{g}, K, D\}, D \subset \mathbb{R}^{n}$, which generates the world function $\sigma\left(x, x^{\prime}\right)$ defined by relation (4.1). Then the $\sigma$-space $V=\{\sigma, D\}$ appears. It will be referred to as $\sigma$-Riemannian space. We are going to compare the concept of collinearity (parallelism) of two vectors in the two spaces.

The world function $\sigma=\sigma\left(x, x^{\prime}\right)$ of both $\sigma$-Riemannian and Riemannian spaces satisfies the system of equations [7] (the paper [7] is hardly available for English speaking reader. Survey of main results of [7] in English may be found in [8], see also [11]).

$$
\begin{gathered}
\sigma_{l} \sigma^{l j^{\prime}} \sigma_{j^{\prime}}=2 \sigma, \quad \sigma\left(x, x^{\prime}\right)=\sigma\left(x^{\prime}, x\right), \\
\sigma(x, x)=0, \quad \operatorname{det}\left\|\sigma_{i \| k}\right\| \neq 0, \\
\operatorname{det}\left\|\sigma_{i k^{\prime}}\right\| \neq 0, \quad \sigma_{i\|k\| l}=0,
\end{gathered}
$$

where the following designations are used

$$
\sigma_{i} \equiv \frac{\partial \sigma}{\partial x^{i}}, \quad \sigma_{i^{\prime}} \equiv \frac{\partial \sigma}{\partial x^{\prime i}}, \quad \sigma_{i k^{\prime}} \equiv \frac{\partial^{2} \sigma}{\partial x^{i} \partial x^{\prime k}}, \quad \sigma^{i k^{\prime}} \sigma_{l k^{\prime}}=\delta_{l}^{i} .
$$

Here the primed index corresponds to the point $x^{\prime}$, and unprimed index corresponds to the point $x$. Symbol $\|$ before index $i$ denotes covariant derivative $\tilde{\nabla}_{i}^{x^{\prime}}$ with respect to $x^{i}\left(\sigma_{k \| i} \equiv \tilde{\nabla}_{i}^{x^{\prime}} \sigma_{k}\right)$. The derivative $\tilde{\nabla}_{i}^{x^{\prime}}$ is the covariant derivative with respect to with $x^{i}$ with the Christoffel symbol

$$
\Gamma_{k l}^{i} \equiv \Gamma_{k l}^{i}\left(x, x^{\prime}\right) \equiv \sigma^{i s^{\prime}} \sigma_{k l s^{\prime}}, \quad \sigma_{k l s^{\prime}} \equiv \frac{\partial^{3} \sigma}{\partial x^{k} \partial x^{l} \partial x^{\prime s}} .
$$

For instance,

$$
\begin{aligned}
G_{i k} \equiv G_{i k}\left(x, x^{\prime}\right) & \equiv \sigma_{i \| k} \equiv \frac{\partial \sigma_{i}}{\partial x^{k}}-\Gamma_{i k}^{l}\left(x, x^{\prime}\right) \sigma_{l} \equiv \frac{\partial \sigma_{i}}{\partial x^{k}}-\sigma_{i k s^{\prime}} \sigma^{l s^{\prime}} \sigma_{l} \\
G_{i k \| l} & \equiv \frac{\partial G_{i k}}{\partial x^{l}}-\sigma_{i l s^{\prime}} \sigma^{j s^{\prime}} G_{j k}-\sigma_{k l s^{\prime}} \sigma^{j s^{\prime}} G_{i j} .
\end{aligned}
$$

Summation from 1 to $n$ is produced over repeated indices. The covariant derivative $\tilde{\nabla}_{i}^{x^{\prime}}$ with respect to $x^{i}$ with the Christoffel symbol $\Gamma_{k l}^{i}\left(x, x^{\prime}\right)$ acts only on the point $x$ and on unprimed indices. It is called the tangent derivative, because it is a covariant derivative in the Euclidean space $E_{\chi^{\prime}}$ which is tangent to the Riemannian space $R_{n}$ at the point $x^{\prime}$. The covariant derivative $\tilde{\nabla}_{i^{\prime}}^{x}$ with respect to $x^{\prime i}$ with the Christoffel symbol $\Gamma_{k^{\prime} l^{\prime}}^{i^{\prime}}\left(x, x^{\prime}\right)$ acts only on the point $x^{\prime}$ and on primed indices. It is a covariant derivative in the Euclidean space $E_{x}$ that is tangent to the $\sigma$-Riemannian space $R_{n}$ at the point $x$ [7].

In general, the world function $\sigma$ carries out the geodesic mapping $G_{x^{\prime}}: R_{n} \rightarrow E_{x^{\prime}}$ of the Riemannian space $R_{n}=\{\mathbf{g}, K, D\}$ on the Euclidean space $E_{x^{\prime}}=\left\{\mathbf{g}, K_{x^{\prime}}, D\right\}$, tangent to $R_{n}=\{\mathbf{g}, K, D\}$ at the point $x^{\prime}$ [7]. This mapping transforms the coordinate system $K$ in $R_{n}$ into the coordinate system $K_{x^{\prime}}$ in $E_{x^{\prime}}$. The mapping is geodesic in the sense that it conserves the lengths of segments of all geodesics, passing through the point of contact $x^{\prime}$ and angles between them at this point.

The tensor $G_{i k}$ defined by (5.4) is the metric tensor at the point $x$ in the tangent Euclidean space $E_{x^{\prime}}$. The covariant derivatives $\tilde{\nabla}_{i}^{x^{\prime}}$ and $\tilde{\nabla}_{k}^{x^{\prime}}$ commute identically, that 
is, $\left(\tilde{\nabla}_{i}^{x^{\prime}} \tilde{\nabla}_{k}^{x^{\prime}}-\tilde{\nabla}_{k}^{x^{\prime}} \tilde{\nabla}_{i}^{x^{\prime}}\right) A_{l s} \equiv 0$, for any tensor $A_{l s}[7]$. This shows that they are covariant derivatives in the flat space $E_{x^{\prime}}$.

The system of equations (5.1) contains only world function $\sigma$ and its derivatives, nevertheless the system of equations (5.1) is not $\sigma$-immanent, because it contains a reference to a coordinate system. It does not contain the metric tensor explicitly. Hence, it is valid for any Riemannian space $R_{n}=\{\mathbf{g}, K, D\}$. All the relations written above are valid as well for the $\sigma$-space $V=\{\sigma, D\}$, provided that the world function $\sigma$ is coupled with the metric tensor by relation (4.1).

The $\sigma$-immanent expression for scalar product $\left(\mathbf{P}_{0} \mathbf{P}_{1} \cdot \mathbf{Q}_{0} \mathbf{Q}_{1}\right)$ of the two vectors $\mathbf{P}_{0} \mathbf{P}_{1}$ and $\mathbf{Q}_{0} \mathbf{Q}_{1}$ in the proper Euclidean space has the form

$$
\left(\mathbf{P}_{0} \mathbf{P}_{1} \cdot \mathbf{Q}_{0} \mathbf{Q}_{1}\right) \equiv \sigma\left(P_{0}, Q_{1}\right)+\sigma\left(Q_{0}, P_{1}\right)-\sigma\left(P_{0}, Q_{0}\right)-\sigma\left(P_{1}, Q_{1}\right) .
$$

This relation can be easily proved as follows.

In the proper Euclidean space three vectors $\mathbf{P}_{0} \mathbf{P}_{1}, \mathbf{P}_{0} \mathbf{Q}_{1}$, and $\mathbf{P}_{1} \mathbf{Q}_{1}$ are coupled by the relation

$$
\left|\mathbf{P}_{1} \mathbf{Q}_{1}\right|^{2}=\left|\mathbf{P}_{0} \mathbf{Q}_{1}-\mathbf{P}_{0} \mathbf{P}_{1}\right|^{2}=\left|\mathbf{P}_{0} \mathbf{P}_{1}\right|^{2}+\left|\mathbf{P}_{0} \mathbf{Q}_{1}\right|^{2}-2\left(\mathbf{P}_{0} \mathbf{P}_{1} \cdot \mathbf{P}_{0} \mathbf{Q}_{1}\right),
$$

where $\left(\mathbf{P}_{0} \mathbf{P}_{1} \cdot \mathbf{P}_{0} \mathbf{Q}_{1}\right)$ denotes the scalar product of two vectors $\mathbf{P}_{0} \mathbf{P}_{1}$ and $\mathbf{P}_{0} \mathbf{Q}_{1}$ in the proper Euclidean space. It follows from (5.6) that

$$
\left(\mathbf{P}_{0} \mathbf{P}_{1} \cdot \mathbf{P}_{0} \mathbf{Q}_{1}\right)=\frac{1}{2}\left\{\left|\mathbf{P}_{0} \mathbf{Q}_{1}\right|^{2}+\left|\mathbf{P}_{0} \mathbf{P}_{1}\right|^{2}-\left|\mathbf{P}_{1} \mathbf{Q}_{1}\right|^{2}\right\} .
$$

Substituting the point $Q_{1}$ by $Q_{0}$ in (5.7), we obtain

$$
\left(\mathbf{P}_{0} \mathbf{P}_{1} \cdot \mathbf{P}_{0} \mathbf{Q}_{0}\right)=\frac{1}{2}\left\{\left|\mathbf{P}_{0} \mathbf{Q}_{0}\right|^{2}+\left|\mathbf{P}_{0} \mathbf{P}_{1}\right|^{2}-\left|\mathbf{P}_{1} \mathbf{Q}_{0}\right|^{2}\right\} .
$$

Subtracting (5.8) from (5.7) and using the properties of the scalar product in the proper Euclidean space, we obtain

$$
\left(\mathbf{P}_{0} \mathbf{P}_{1} \cdot \mathbf{Q}_{0} \mathbf{Q}_{1}\right)=\frac{1}{2}\left\{\left|\mathbf{P}_{0} \mathbf{Q}_{1}\right|^{2}+\left|\mathbf{Q}_{0} \mathbf{P}_{1}\right|^{2}-\left|\mathbf{P}_{0} \mathbf{Q}_{0}\right|^{2}-\left|\mathbf{P}_{1} \mathbf{Q}_{1}\right|^{2}\right\} .
$$

Taking into account that $\left|\mathbf{P}_{0} \mathbf{Q}_{1}\right|^{2}=2 \sigma\left(P_{0}, Q_{1}\right)$, we obtain the relation (5.5) from relation (5.9).

The two vectors $\mathbf{P}_{0} \mathbf{P}_{1}$ and $\mathbf{Q}_{0} \mathbf{Q}_{1}$ are collinear $\mathbf{P}_{0} \mathbf{P}_{1} \| \mathbf{Q}_{0} \mathbf{Q}_{1}$ (parallel or antiparallel), provided that $\cos ^{2} \theta=1$, where $\theta$ is the angle between the vectors $\mathbf{P}_{0} \mathbf{P}_{1}$ and $\mathbf{Q}_{0} \mathbf{Q}_{1}$. Taking into account that

$$
\cos ^{2} \theta=\frac{\left(\mathbf{P}_{0} \mathbf{P}_{1} \cdot \mathbf{Q}_{0} \mathbf{Q}_{1}\right)^{2}}{\left(\mathbf{P}_{0} \mathbf{P}_{1} \cdot \mathbf{P}_{0} \mathbf{P}_{1}\right)\left(\mathbf{Q}_{0} \mathbf{Q}_{1} \cdot \mathbf{Q}_{0} \mathbf{Q}_{1}\right)}=\frac{\left(\mathbf{P}_{0} \mathbf{P}_{1} \cdot \mathbf{Q}_{0} \mathbf{Q}_{1}\right)^{2}}{\left|\mathbf{P}_{0} \mathbf{P}_{1}\right|^{2} \cdot\left|\mathbf{Q}_{0} \mathbf{Q}_{1}\right|^{2}},
$$

we obtain the following $\sigma$-immanent condition of the two vectors collinearity

$$
\mathbf{P}_{0} \mathbf{P}_{1} \| \mathbf{Q}_{0} \mathbf{Q}_{1}:\left(\mathbf{P}_{0} \mathbf{P}_{1} \cdot \mathbf{Q}_{0} \mathbf{Q}_{1}\right)^{2}=\left|\mathbf{P}_{0} \mathbf{P}_{1}\right|^{2} \cdot\left|\mathbf{Q}_{0} \mathbf{Q}_{1}\right|^{2} .
$$

The collinearity condition (5.11) is $\sigma$-immanent, because by means of (5.5) it can be written in terms of the $\sigma$-function only. Thus, this relation describes the vectors collinearity in the case of arbitrary $\sigma$-space. 
We describe this relation for the case of $\sigma$-Riemannian geometry. Let the coordinates of points $P_{0}, P_{1}, Q_{0}, Q_{1}$ be respectively $x, x+d x, x^{\prime}$, and $x^{\prime}+d x^{\prime}$. Then writing (5.5) and expanding it over $d x$ and $d x^{\prime}$, we obtain

$$
\begin{aligned}
\left(\mathbf{P}_{0} \mathbf{P}_{1} \cdot \mathbf{Q}_{0} \mathbf{Q}_{1}\right) \equiv & \sigma\left(x, x^{\prime}+d x^{\prime}\right)+\sigma\left(x^{\prime}, x+d x\right)-\sigma\left(x, x^{\prime}\right)-\sigma\left(x+d x, x^{\prime}+d x^{\prime}\right) \\
= & \sigma_{l^{\prime}} d x^{\prime l^{\prime}}+\frac{1}{2} \sigma_{l^{\prime}, s^{\prime}} d x^{\prime l^{\prime}} d x^{\prime s^{\prime}}+\sigma_{i} d x^{i}+\frac{1}{2} \sigma_{i, k} d x^{i} d x^{k} \\
& -\sigma_{i} d x^{i}-\sigma_{l^{\prime}} d x^{\prime l^{\prime}}-\frac{1}{2} \sigma_{i, k} d x^{i} d x^{k}-\sigma_{i, l^{\prime}} d x^{i} d x^{\prime l^{\prime}}-\frac{1}{2} \sigma_{l^{\prime}, s^{\prime}} d x^{\prime l^{\prime}} d x^{\prime s^{\prime}} \\
\left(\mathbf{P}_{0} \mathbf{P}_{1} \cdot \mathbf{Q}_{0} \mathbf{Q}_{1}\right)= & -\sigma_{i, l^{\prime}} d x^{i} d x^{\prime l^{\prime}}=-\sigma_{i l^{\prime}} d x^{i} d x^{\prime l^{\prime}} .
\end{aligned}
$$

Here the comma means differentiation. For instance, $\sigma_{i, k} \equiv \partial \sigma_{i} / \partial x^{k}$. We obtain for $\left|\mathbf{P}_{0} \mathbf{P}_{1}\right|^{2}$ and $\left|\mathbf{Q}_{0} \mathbf{Q}_{1}\right|^{2}$

$$
\left|\mathbf{P}_{0} \mathbf{P}_{1}\right|^{2}=g_{i k} d x^{i} d x^{k}, \quad\left|\mathbf{Q}_{0} \mathbf{Q}_{1}\right|^{2}=g_{l^{\prime} s^{\prime}} d x^{\prime l^{\prime}} d x^{\prime s^{\prime}},
$$

where $g_{i k}=g_{i k}(x)$ and $g_{l^{\prime} s^{\prime}}=g_{l^{\prime} s^{\prime}}\left(x^{\prime}\right)$. Then the collinearity condition (5.11) is written in the form

$$
\left(\sigma_{i l^{\prime}} \sigma_{k s^{\prime}}-g_{i k} g_{l^{\prime} s^{\prime}}\right) d x^{i} d x^{k} d x^{\prime l^{\prime}} d x^{\prime s^{\prime}}=0 .
$$

Take into account that in the Riemannian space, the metric tensor $g_{l^{\prime}} s^{\prime}$ at the point $x^{\prime}$ can be expressed via the world function $\sigma$ of points $x, x^{\prime}$ by means of relation [7]

$$
g_{l^{\prime} s^{\prime}}=\sigma_{i l^{\prime}} G^{i k} \sigma_{k s^{\prime}}, \quad g^{l^{\prime} s^{\prime}}=\sigma^{i l^{\prime}} G_{i k} \sigma^{k s^{\prime}}
$$

where the tensor $G_{i k}$ is defined by relation (5.4), and $G^{i k}$ is defined by the relation

$$
G^{i l} G_{l k}=\delta_{k}^{i}
$$

Substituting the first relation (5.4) in (5.14) and using designation

$$
u_{i}=-\sigma_{i l^{\prime}} d x^{\prime l^{\prime}}, \quad u^{i}=G^{i k} u_{k}=-\sigma^{i l^{\prime}} g_{l^{\prime} s^{\prime}} d x^{s^{\prime}}
$$

we obtain

$$
\left(\delta_{i}^{l} \delta_{k}^{s}-g_{i k} G^{l s}\right) u_{l} u_{s} d x^{i} d x^{k}=0
$$

The vector $u_{i}$ is the vector $d x_{i^{\prime}}^{\prime}=g_{i^{\prime} k^{\prime}} d x^{\prime k^{\prime}}$ transported parallelly from the point $x^{\prime}$ to the point $x$ in the Euclidean space $E_{x^{\prime}}$ tangent to the Riemannian space $R_{n}$. Indeed,

$$
u_{i}=-\sigma_{i l^{\prime}} g^{l^{\prime} s^{\prime}} d x_{s}^{\prime}, \quad \tilde{\nabla}_{k}^{x^{\prime}}\left(-\sigma_{i l^{\prime}} \mathcal{g}^{l^{\prime} s^{\prime}}\right) \equiv 0, \quad i, k=1,2, \ldots, n
$$

and tensor $-\sigma_{i l^{\prime}} g^{l^{\prime} s^{\prime}}$ is the operator of the parallel transport in $E_{\chi^{\prime}}$, because

$$
\left[-\sigma_{i l^{\prime}} \mathcal{g}^{l^{\prime} s^{\prime}}\right]_{x=x^{\prime}}=\delta_{i^{\prime}}^{s^{\prime}}
$$


and the tangent derivative of this operator is equal to zero identically. For the same reason, that is, because of

$$
\left[\sigma^{i l^{\prime}} g_{l^{\prime} s^{\prime}} \sigma^{k s^{\prime}}\right]_{x=x^{\prime}}=g^{i^{\prime} k^{\prime}}, \quad \tilde{\nabla}_{s}^{x^{\prime}}\left(\sigma^{i l^{\prime}} g_{l^{\prime} s^{\prime}} \sigma^{k s^{\prime}}\right) \equiv 0
$$

$G^{i k}=\sigma^{i l^{\prime}} g_{l^{\prime} s^{\prime}} \sigma^{k s^{\prime}}$ is the contravariant metric tensor in $E_{x^{\prime}}$, at the point $x$.

Relation (5.18) contains vectors at the point $x$ only. At fixed $u_{i}=-\sigma_{i l^{\prime}} d x^{\prime l^{\prime}}$ it describes a collinearity cone, that is, a cone of infinitesimal vectors $d x^{i}$ at the point $x$ parallel to the vector $d x^{\prime i^{\prime}}$ at the point $x^{\prime}$. Under some conditions, the collinearity cone can degenerates into a line. In this case there is only one direction parallel to the fixed vector $u^{i}$. We investigate when this situation takes place.

At the point $x$ two metric tensors $g_{i k}$ and $G_{i k}$ are connected by the relation [7]

$$
G_{i k}\left(x, x^{\prime}\right)=g_{i k}(x)+\int_{x}^{x^{\prime}} F_{i k j^{\prime \prime} s^{\prime \prime}}\left(x, x^{\prime \prime}\right) \sigma^{j^{\prime \prime}}\left(x, x^{\prime \prime}\right) d x^{\prime \prime} s^{\prime \prime},
$$

where, according to [7],

$$
\sigma^{i^{\prime}}=\sigma^{l i^{\prime}} \sigma_{l}=G^{l^{\prime} i^{\prime}} \sigma_{l^{\prime}}=g^{l^{\prime} i^{\prime}} \sigma_{l^{\prime}}
$$

Integration does not depend on the path, because it is produced in the Euclidean space $E_{\chi^{\prime}}$. The two-point tensor $F_{i l k^{\prime} j^{\prime}}=F_{i l k^{\prime} j^{\prime}}\left(x, x^{\prime}\right)$ is the two-point curvature tensor, defined by the relation

$$
F_{i l k^{\prime} j^{\prime}}=\sigma_{i l j^{\prime} \| k^{\prime}}=\sigma_{i l j^{\prime}, k^{\prime}}-\sigma_{s j^{\prime} k^{\prime}} \sigma^{s m^{\prime}} \sigma_{i l m^{\prime}}=\sigma_{i \mid l\left\|k^{\prime}\right\| j^{\prime}},
$$

where one vertical stroke denotes usual covariant derivative and two vertical strokes denote tangent derivative. The two-point curvature tensor $F_{i l k^{\prime} j^{\prime}}$ has the following symmetry properties

$$
F_{i l k^{\prime} j^{\prime}}=F_{l i k^{\prime} j^{\prime}}=F_{i l j^{\prime} k^{\prime}}, \quad F_{i l k^{\prime} j^{\prime}}\left(x, x^{\prime}\right)=F_{k^{\prime} j^{\prime} i l}\left(x^{\prime}, x\right) .
$$

It is connected with the one-point Riemann-Christoffel curvature tensor $r_{i l j k}$ by means of the relations

$$
r_{i l j k}=\left[F_{i k j^{\prime} l^{\prime}}-F_{i j k^{\prime} l^{\prime}}\right]_{x^{\prime}=x}=f_{i k j l}-f_{i j k l}, \quad f_{i k l j}=\left[F_{i k j^{\prime} l^{\prime}}\right]_{x^{\prime}=x} .
$$

In the Euclidean space, the two-point curvature tensor $F_{i l k^{\prime} j^{\prime}}$ vanishes as well as the Riemann-Christoffel curvature tensor $r_{i l j k}$.

We introduce the designation

$$
\Delta_{i k}=\Delta_{i k}\left(x, x^{\prime}\right)=\int_{x}^{x^{\prime}} F_{i k j^{\prime \prime} s^{\prime \prime}}\left(x, x^{\prime \prime}\right) \sigma^{j^{\prime \prime}}\left(x, x^{\prime \prime}\right) d x^{\prime \prime} s^{\prime \prime}
$$

and choose the geodesic $\mathscr{L}_{x x^{\prime}}$ as the path of integration. It is described by the relation

$$
\sigma_{i}\left(x, x^{\prime \prime}\right)=\tau \sigma_{i}\left(x, x^{\prime}\right)
$$

which determines $x^{\prime \prime}$ as a function of parameter $\tau$. Differentiating with respect to $\tau$, we obtain

$$
\sigma_{i k^{\prime \prime}}\left(x, x^{\prime \prime}\right) d x^{\prime \prime k^{\prime \prime}}=\sigma_{i}\left(x, x^{\prime}\right) d \tau \text {. }
$$


Resolving (5.29) with respect to $d x^{\prime \prime}$ and substituting in (5.27), we obtain

$$
\Delta_{i k}\left(x, x^{\prime}\right)=\sigma_{l}\left(x, x^{\prime}\right) \sigma_{p}\left(x, x^{\prime}\right) \int_{0}^{1} F_{i k j^{\prime \prime} s^{\prime \prime}}\left(x, x^{\prime \prime}\right) \sigma^{l j^{\prime \prime}}\left(x, x^{\prime \prime}\right) \sigma^{p s^{\prime \prime}}\left(x, x^{\prime \prime}\right) \tau d \tau,
$$

where $x^{\prime \prime}$ is determined by (5.28) as a function of $\tau$. We set

$$
F_{i k}^{l p}\left(x, x^{\prime}\right)=F_{i k j^{\prime} s^{\prime}}\left(x, x^{\prime}\right) \sigma^{l j^{\prime}}\left(x, x^{\prime}\right) \sigma^{p s^{\prime}}\left(x, x^{\prime}\right)
$$

then

$$
\begin{gathered}
G_{i k}\left(x, x^{\prime}\right)=g_{i k}(x)+\Delta_{i k}\left(x, x^{\prime}\right) \\
\Delta_{i k}\left(x, x^{\prime}\right)=\sigma_{l}\left(x, x^{\prime}\right) \sigma_{p}\left(x, x^{\prime}\right) \int_{0}^{1} F_{i k}^{l p}\left(x, x^{\prime \prime}\right) \tau d \tau .
\end{gathered}
$$

Substituting $g_{i k}$ from (5.32) in (5.18), we obtain

$$
\left(\delta_{i}^{l} \delta_{k}^{s}-G^{l s}\left(G_{i k}-\Delta_{i k}\right)\right) u_{l} u_{s} d x^{i} d x^{k}=0 .
$$

And we look for solutions to the equation in the form of expansion

$$
d x^{i}=\alpha u^{i}+v^{i}, \quad G_{i k} u^{i} v^{k}=0 .
$$

Substituting (5.35) in (5.34), we obtain an equation for $v^{i}$

$$
G_{l s} u^{l} u^{s}\left[G_{i k} v^{i} v^{k}-\Delta_{i k}\left(\alpha u^{i}+v^{i}\right)\left(\alpha u^{k}+v^{k}\right)\right]=0 .
$$

If the $\sigma$-Riemannian space $V=\{\sigma, D\}$ is $\sigma$-Euclidean, then as it follows from (5.33) $\Delta_{i k}=0$. If $V=\{\sigma, D\}$ is the proper $\sigma$-Euclidean space, $G_{l s} u^{l} u^{s} \neq 0$, and we obtain two equations to determine $v^{i}$

$$
G_{i k} v^{i} v^{k}=0, \quad G_{i k} u^{i} v^{k}=0 .
$$

The only solution

$$
v^{i}=0, \quad d x^{i}=\alpha u^{i}, \quad i=1,2, \ldots, n
$$

of (5.36) is a solution of (5.34), where $\alpha$ is an arbitrary constant. In the proper Euclidean geometry, the collinearity cone always degenerates into a line.

Let now the space $V=\{\sigma, D\}$ be the $\sigma$-pseudo-Euclidean space of index 1, and the vector $u^{i}$ be timelike, that is, $G_{i k} u^{i} u^{k}>0$. Then (5.37) also have the solution (5.38). If the vector $u^{i}$ is spacelike, $G_{i k} u^{i} u^{k}<0$, then the two equations (5.37) have a nontrivial solution, and the collinearity cone does not degenerate into a line. The collinearity cone is a section of the light cone $G_{i k} v^{i} v^{k}=0$ by the plane $G_{i k} u^{i} v^{k}=0$. If the vector $u^{i}$ is null, $G_{i k} u^{i} u^{k}=0$, then (5.36) reduces to the form

$$
G_{i k} u^{i} u^{k}=0, \quad G_{i k} u^{i} v^{k}=0 .
$$

In this case (5.38) is a solution, but besides, there are spacelike vectors $v^{i}$ that are orthogonal to null vector $u^{i}$ and the collinearity cone does not degenerate into a line. 
In the case of the proper $\sigma$-Riemannian space $G_{i k} u^{i} u^{k}>0$, and (5.36) reduces to the form

$$
G_{i k} v^{i} v^{k}-\Delta_{i k}\left(\alpha u^{i}+v^{i}\right)\left(\alpha u^{k}+v^{k}\right)=0 .
$$

In this case, $\Delta_{i k} \neq 0$ in general and the collinearity cone does not degenerate. The quantity $\Delta_{i k}$ depends on the curvature and on the distance between the points $x$ and $x^{\prime}$. The greater are the space curvature and the distance $\rho\left(x, x^{\prime}\right)$, the greater is the collinearity cone aperture.

In the curved proper $\sigma$-Riemannian space, there is an interesting special case where the collinearity cone degenerates. In any $\sigma$-Riemannian space, the following equality takes place [7]

$$
G_{i k} \sigma^{k}=g_{i k} \sigma^{k}, \quad \sigma^{k} \equiv g^{k l} \sigma_{l} .
$$

Then it follows from (5.32) that

$$
\Delta_{i k} \sigma^{k}=0
$$

It means that in the case where the vector $u^{i}$ is directed along the geodesic, connecting points $x$ and $x^{\prime}$, that is, $u^{i}=\beta \sigma^{i}$, (5.40) reduces to the form

$$
\left(G_{i k}-\Delta_{i k}\right) v^{i} v^{k}=0, \quad u^{i}=\beta \sigma^{i} .
$$

If $\Delta_{i k}$ is small enough when compared to $G_{i k}$, then the eigenvalues of the matrix $G_{i k}-\Delta_{i k}$ have the same sign, as those of the matrix $G_{i k}$. In this case (5.43) has the only solution (5.38), and the collinearity cone degenerates.

6. Pseudo-Riemannian geometry and one-dimensionality of the first order tubes. In general, the collinearity cone does not degenerate even in a flat Riemannian space if it is not a proper Riemannian one. We have seen this in the previous section for the collinearity cone of infinitesimal vectors (relations (5.39)). Now we consider the special case of finite vectors in flat Riemannian space and, in particular, in the Minkowski space.

We consider the $n$-dimensional pseudo-Euclidean space $E_{n}=\left\{\mathbf{g}_{1}, K, \mathbb{R}^{n}\right\}$ of the index $1, \mathbf{g}_{1}=\operatorname{diag}\{1,-1,-1, \ldots,-1\}$ to be a special case of $n$-dimensional pseudoRiemannian space. The world function is defined by relation (2.25)

$$
\sigma_{1}\left(x, x^{\prime}\right)=\frac{1}{2} \sum_{i, k=1}^{n} g^{i k}\left(x_{i}-x_{i}^{\prime}\right)\left(x_{k}-x_{k}^{\prime}\right), \quad g^{i k}=\operatorname{diag}\{1,-1,-1, \ldots,-1\} .
$$

The geodesic $\mathscr{L}_{y y^{\prime}}$ is a straight line and is considered in the pseudo-Euclidean geometry to be the first order NGO, determined by two points $y$ and $y^{\prime}$, that is, it is determined by two points $y$ and $y^{\prime}$ and by the geometry itself

$$
\mathscr{L}_{y y^{\prime}}: x^{i}=\left(y^{i}-y^{\prime i}\right) \tau, \quad i=1,2, \ldots, n, \tau \in \mathbb{R} .
$$

The geodesic $\mathscr{L}_{y y^{\prime}}$ is called timelike if $\sigma_{1}\left(y, y^{\prime}\right)>0$, and it is called spacelike if $\sigma_{1}\left(y, y^{\prime}\right)<0$. The geodesic $\mathscr{L}_{y y^{\prime}}$ is called null if $\sigma_{1}\left(y, y^{\prime}\right)=0$. 
The pseudo-Euclidean space $E_{n}=\left\{\mathbf{g}_{1}, K, \mathbb{R}^{n}\right\}$ generates the $\sigma$-space $V=\left\{\sigma_{1}, \mathbb{R}^{n}\right\}$, where the world function $\sigma_{1}$ is defined by relation (6.1). The $\sigma$-space $V=\left\{\sigma_{1}, \mathbb{R}^{n}\right\}$ will be referred to as $\sigma$-pseudo-Euclidean space (special case of $\sigma$-Riemannian space). The first order tube (NGO) $\mathscr{T}_{x, x^{\prime}}$ in the $\sigma$-pseudo-Euclidean space $V=\left\{\sigma_{1}, \mathbb{R}^{n}\right\}$ is defined by relation (2.22)

$$
\begin{gathered}
\mathscr{T}\left(x, x^{\prime}\right) \equiv \mathcal{T}_{x x^{\prime}}=\left\{r \mid F_{2}\left(x, x^{\prime}, r\right)=0\right\}, \quad \sigma_{1}\left(x, x^{\prime}\right) \neq 0, \quad x, x^{\prime}, r \in \mathbb{R}^{n}, \\
F_{2}\left(x, x^{\prime}, r\right)=\left|\begin{array}{ll}
\left(x_{i}^{\prime}-x_{i}\right)\left(x^{\prime}-x^{i}\right) & \left(x_{i}^{\prime}-x_{i}\right)\left(r^{i}-x^{i}\right) \\
\left(r_{i}-x_{i}\right)\left(x^{\prime}-x^{i}\right) & \left(r_{i}-x_{i}\right)\left(r^{i}-x^{i}\right)
\end{array}\right| .
\end{gathered}
$$

In other words, $\mathscr{T}_{x x^{\prime}}=\mathscr{T}\left(x, x^{\prime}\right)$ is also NGO, because it is also determined by two points $x$ and $x^{\prime}$ and the T-geometry itself. The solution of (6.3) and (6.4) yields

$$
\begin{gathered}
\mathscr{T}_{x x^{\prime}}=\left\{r \mid \bigcup_{y \in \mathbb{R}^{n}} \bigcup_{\tau \in \mathbb{R}} r=\left(x^{\prime}-x\right) \tau+y-x \wedge \Gamma\left(x, x^{\prime}, y\right)=0 \wedge \Gamma(x, y, y)=0\right\}, \\
x, x^{\prime}, y, r \in \mathbb{R}^{n},
\end{gathered}
$$

where $\Gamma\left(x, x^{\prime}, y\right)=\left(x_{i}^{\prime}-x_{i}\right)\left(y^{i}-x^{i}\right)$ is the scalar product of the vectors $\overrightarrow{x y}$ and $\overrightarrow{x x^{\prime}}$ defined by relation (2.7). In the case of timelike vector $\overrightarrow{x x^{\prime}}$, when $\sigma_{1}\left(x, x^{\prime}\right)>0$, there is a unique null vector $\overrightarrow{x y}=\overrightarrow{x x}=\overrightarrow{0}$ which is orthogonal to the vector $\overrightarrow{x x^{\prime}}$. In this case, the $(n-1)$-dimensional surface $\mathscr{T}_{x x^{\prime}}$ degenerates into the one-dimensional straight

$$
\mathscr{T}_{x x^{\prime}}=\left\{r \mid \bigcup_{\tau \in \mathbb{R}} r=\left(x^{\prime}-x\right) \tau\right\}, \quad \sigma_{1}\left(x, x^{\prime}\right)>0, \quad x, x^{\prime}, r \in \mathbb{R}^{n} .
$$

Thus, for timelike vector $\overrightarrow{x x^{\prime}}$ the first order tube $\mathscr{T}_{x x^{\prime}}$ coincides with the geodesic $\mathscr{L}_{x x^{\prime}}$. In the case of spacelike vector $\overrightarrow{x x^{\prime}}$ the $(n-1)$-dimensional tube $\mathscr{T}_{x x^{\prime}}$ contains the onedimensional geodesic $\mathscr{L}_{x x^{\prime}}$ of the pseudo-Euclidean space $E_{n}=\left\{\mathbf{g}_{1}, K, \mathbb{R}^{n}\right\}$. The difference between $\mathscr{L}_{x x^{\prime}}$ and $\mathscr{T}_{x x^{\prime}}$ is conditioned by different definitions of collinearity in the pseudo-Euclidean space and in the $\sigma$-pseudo-Euclidean one. In the pseudoEuclidean space $E_{n}=\left\{\mathbf{g}_{1}, K, \mathbb{R}^{n}\right\}$, the two vectors are collinear if their rectilinear coordinates are proportional, whereas in the $\sigma$-pseudo-Euclidean space $V=\left\{\sigma_{1}, \mathbb{R}^{n}\right\}$ they are collinear, provided that they satisfy relation (2.7). For instance, for $n=4$ two spacelike vectors $\mathbf{a}=\{0,1,0,0\}$ and $\mathbf{b}=\{1,1,1,0\}$ are collinear in $V=\left\{\sigma_{1}, \mathbb{R}^{4}\right\}$ and not collinear in $E_{4}=\left\{\mathbf{g}_{1}, K, \mathbb{R}^{4}\right\}$. The definition of collinearity in $V=\left\{\sigma_{1}, \mathbb{R}^{4}\right\}$ is $\sigma$ immanent, whereas this definition in the pseudo-Euclidean space $E_{n}=\left\{\mathbf{g}_{1}, K, \mathbb{R}^{n}\right\}$ is not $\sigma$-immanent, because it contains a reference to a coordinate system. From logical viewpoint the $\sigma$-immanent definition is more perfect, because it does not contain nongeometrical concepts.

For $n=4$, the pseudo-Euclidean space $E_{4}=\left\{\mathbf{g}_{1}, K, \mathbb{R}^{4}\right\}$, and the $\sigma$-pseudo-Euclidean space $V=\left\{\sigma_{1}, \mathbb{R}^{4}\right\}$ describe the real space-time (the Minkowski space), using two different geometrical conceptions. In this case the first order NGOs associate with real physical objects-free particles. For usual free particles, whose world line is timelike straight, both conceptions give the same result. In general, the pseudo-Euclidean space $E_{4}=\left\{\mathbf{g}_{1}, K, \mathbb{R}^{4}\right\}$ (Riemannian conception) admits the existence of hypothetical particles (taxyons), moving with the speed, which is more than the speed of the light. It is 
usually supposed that a taxyon is described by the spacelike straight $\mathscr{L}_{x x^{\prime}}$. Taxyons failed to be discovered experimentally.

The $\sigma$-pseudo-Euclidean space $V=\left\{\sigma_{1}, \mathbb{R}^{4}\right\}$ admits the existence of taxyons (the particles, moving with the speed, which is more than the speed of the light) to be described only by the three-dimensional surface $\mathcal{T}_{x x^{\prime}}$. It explains the failure of their discovery by the fact that taxyons are looked for in the form of spacelike straights (but not in the form of three-dimensional surfaces). It is not clear what means from physical viewpoint the statement: the particle moving with the speed, which is more than the speed of the light, is described by means of three-dimensional surface. Maybe, here the following association is useful.

The particles, whose velocity is more than the speed of the light in the matter (but not in the vacuum), are known. These are the so-called Cherenkov particles. A charged Cherenkov particle radiates an electromagnetic emanation in some directions, forming a cone in the three-dimensional space. The set of all events, describing the motion of a Cherenkov particle and that of photons radiated by it, forms a three-dimensional surface $\mathscr{S}_{3}$ in the four-dimensional space-time. The shape of $\mathscr{S}_{3}$ resembles the shape of the surface $\mathscr{T}_{x x^{\prime}}$ with spacelike vector $\overrightarrow{x x^{\prime}}$. Indeed, the surface $\mathscr{T}_{3}$ may be considered as a set of two-dimensional sections $\mathscr{S}_{y}$ of the light cones $\mathscr{T}_{y}$ with the vertex $y \in \mathscr{L}_{x x^{\prime}}$, where $\mathscr{L}_{x x^{\prime}}$ is the world line of the Cherenkov particle. The two-dimensional sections $\mathscr{S}_{y}$ of the light cone $\mathcal{T}_{y}$ appear as a result of intersection of the $\mathcal{T}_{y}$ with some threedimensional plane $\mathscr{L}_{y}$, passing through the point $y$. The direction of the normal vector to $\mathscr{L}_{y}$ depends on the relative velocity of the Cherenkov particle.

A similar picture arises in the case of the surface $\mathcal{T}_{x x^{\prime}}$ with the spacelike vector $\overrightarrow{x x^{\prime}}$. The first order tube $\mathscr{T}_{x x^{\prime}}$ may be considered to be consisting of sections $\mathscr{S}_{y}=$ $\mathscr{T}_{y} \cap \mathscr{L}_{y x x^{\prime}}, y \in \mathscr{L}_{x x^{\prime}}$

$$
\mathscr{T}_{x x^{\prime}}=\bigcup_{y \in \mathscr{L}_{x x^{\prime}}} \mathscr{T}_{y} \cap \mathscr{L}_{y x x^{\prime}},
$$

where $\mathscr{T}_{x}$ is the zeroth order tube (light cone)

$$
\begin{aligned}
\mathscr{T}_{x} & =\{r \mid \sigma(x, r)=0\}, \quad x, r \in \mathbb{R}^{4}, \\
\mathscr{L}_{y x x^{\prime}}=\{r \mid(\overrightarrow{y r} \cdot \overrightarrow{y x}) & \left.=0 \wedge\left(\overrightarrow{y r} \cdot \overrightarrow{y x^{\prime}}\right)=0 \wedge y \in \mathscr{L}_{x x^{\prime}}\right\}, \quad x, x^{\prime}, y, r \in \mathbb{R}^{4} .
\end{aligned}
$$

Expressions (6.7) and (6.8) are valid in $V=\left\{\sigma_{1}, \mathbb{R}^{4}\right\}$ for timelike and spacelike vector $\overrightarrow{x x^{\prime}}$, but for timelike $\overrightarrow{x x^{\prime}}$ the set $\mathscr{T}_{y} \cap \mathscr{L}_{y x x^{\prime}}=\{y\}$, and relation (6.7) stops to be interesting. Expression (6.7) recalls the corresponding relation for Cherenkov particle. But for the Cherenkov particle the vector $\overrightarrow{x x^{\prime}}$ is timelike although it moves with the speed which is moor than the speed of the light in the matter, whereas for a taxyon the vector $\overrightarrow{x x^{\prime}}$ is spacelike.

7. Discussion. Thus, we see that in the $\sigma$-Riemannian geometry at the point $x$ there are many vectors parallel to a given vector at the point $x^{\prime}$. This set of parallel vectors is described by the collinearity cone. The degeneration of the collinearity cone into a line, when there is only one direction, parallel to the given direction, is an exception rather than a rule, although in the proper Euclidean geometry this degeneration takes place always. Nonuniformity of space destroys the collinearity cone degeneration. 
In the proper Riemannian geometry, where the world function satisfies system (5.1), we succeeded in conserving this degeneration to the direction along the geodesic, connecting points $x$ and $x^{\prime}$. This circumstance is very important for the degeneration of the first order NGOs into geodesic, because the degeneration of NGOs is connected closely with the collinearity cone degeneration.

Indeed, the definition of the first order tube (2.22), or (6.3) may be written also in the form

$$
\mathscr{T}\left(\mathscr{P}^{1}\right) \equiv \mathscr{T}_{P_{0} P_{1}}=\left\{R \mid \mathbf{P}_{0} \mathbf{P}_{1} \| \mathbf{P}_{0} \mathbf{R}\right\}, \quad P_{0}, P_{1}, R \in \Omega,
$$

where collinearity $\mathbf{P}_{0} \mathbf{P}_{1} \| \mathbf{P}_{0} \mathbf{R}$ of two vectors $\mathbf{P}_{0} \mathbf{P}_{1}$ and $\mathbf{P}_{0} \mathbf{R}$ is defined by the $\sigma$ immanent relation (5.11) which can be written in the form

$$
\mathbf{P}_{0} \mathbf{P}_{1} \| \mathbf{P}_{0} \mathbf{R}: F_{2}\left(P_{0}, P_{1}, R\right)=\left|\begin{array}{cc}
\left(\mathbf{P}_{0} \mathbf{P}_{1} \cdot \mathbf{P}_{0} \mathbf{P}_{1}\right) & \left(\mathbf{P}_{0} \mathbf{P}_{1} \cdot \mathbf{P}_{0} \mathbf{R}\right) \\
\left(\mathbf{P}_{0} \mathbf{R} \cdot \mathbf{P}_{0} \mathbf{P}_{1}\right) & \left(\mathbf{P}_{0} \mathbf{R} \cdot \mathbf{P}_{0} \mathbf{R}\right)
\end{array}\right|=0 .
$$

Form (7.1) of the first order tube definition allows us to define the first order tube $\mathscr{T}\left(P_{0}, P_{1} ; Q_{0}\right)$ passing through the point $Q_{0}$ collinear to the given vector $\mathbf{P}_{0} \mathbf{P}_{1}$. This definition has the $\sigma$-immanent form

$$
\mathscr{T}\left(P_{0}, P_{1} ; Q_{0}\right)=\left\{R \mid \mathbf{P}_{0} \mathbf{P}_{1} \| \mathbf{Q}_{0} \mathbf{R}\right\}, \quad P_{0}, P_{1}, Q_{0}, R \in \Omega,
$$

where collinearity $\mathbf{P}_{0} \mathbf{P}_{1} \| \mathbf{Q}_{0} \mathbf{R}$ of the two vectors $\mathbf{P}_{0} \mathbf{P}_{1}$ and $\mathbf{Q}_{0} \mathbf{R}$ is defined by the $\sigma$ immanent relations (5.9) and (5.3). In the proper Euclidean space, tube (7.3) degenerates into the straight line passing through point $Q_{0}$ collinear to the given vector $\mathbf{P}_{0} \mathbf{P}_{1}$.

We define set $\omega_{Q_{0}}=\left\{\mathbf{Q}_{0} \mathbf{Q} \mid Q \in \Omega\right\}$ of vectors $\mathbf{Q}_{0} \mathbf{Q}$. Then

$$
\mathscr{C}\left(P_{0}, P_{1} ; Q_{0}\right)=\left\{\mathbf{Q}_{0} \mathbf{Q} \mid Q \in \mathscr{T}\left(P_{0}, P_{1} ; Q_{0}\right)\right\} \subset \omega_{Q_{0}}
$$

is the collinearity cone of vectors $\mathbf{Q}_{0} \mathbf{Q}$ collinear to vector $\mathbf{P}_{0} \mathbf{P}_{1}$. Thus, the onedimensionality of the first order tubes and the collinearity cone degeneration are a connected phenomena.

In the Riemannian geometry, the very special property of the proper Euclidean geometry (the collinearity cone degeneration) is considered to be a property of any geometry and is extended to the case of Riemannian geometry. The line $\mathscr{L}$, defined as a continuous mapping (1.4), is considered to be the most important geometric object. This object is considered to be more important than the metric, and the metric in the Riemannian geometry is defined in terms of the shortest lines. The use of line as a basic concept of geometry is inadequate for the description of geometry and poses problems, which appears to be artificial.

First, the extension of curves introduces nonlocal features in the geometry description. Nonlocality of description manifests itself

(1) in the violation of isometrical embeddability of nonconvex regions in the space, from which they are cut,

(2) in the violation of absolute parallelism of vectors at different points of space. 
These unnatural properties of Riemannian geometry are corollaries of the metric definition via concept of a curve. In $\sigma$-Riemannian geometry, such properties of Euclidean geometry as absolute parallelism and isometrical embeddability of nonconvex regions conserve completely. All this is a manifestation of the negation of nondegeneracy as a natural property of geometry. But we fail to remove nondegeneracy of nonuniform geometry. It exists for spacelike vectors even in the Minkowski geometry.

As far as we cannot remove nondegeneracy from Riemannian geometry, it seems reasonable to recognize that the nondegeneracy is a natural geometric property, and T-geometric conception is more perfect than the Riemannian conception of geometry. A corollary of this conclusion is a reconstruction of local description and absolute parallelism (the last may be useful for formulation of integral conservation laws in a curved space-time). Besides, the T-geometric conception is essentially simpler than the Riemannian one. It has simpler structure and uses simpler method of description. The fundamental mapping (2.14), introducing the multivector in Tgeometry is essentially simpler than fundamental mapping (1.4), introducing the curve in Riemannian geometry. Mapping (2.14) deals with finite objects. It does not contain any reference to limiting processes nor limits whose existence should be provided. Finally, the T-geometry is not sensitive to, whether the real space-time is continuous or only fine-grained. This is important also, because it seems not to be tested experimentally.

\section{REFERENCES}

[1] A. D. Alexandrov, Internal Geometry of Convex Surfaces, GITTL, Moscow, 1948 (Russian).

[2] A. D. Alexandrov, V. N. Berestovski, and I. G. Nikolayev, Generalized Riemannian spaces, Uspekhi Mat. Nauk 41 (1986), no. 3(249), 3-42 (Russian).

[3] L. M. Blumenthal, Theory and Applications of Distance Geometry, Clarendon Press, Oxford, 1953.

[4] Y. Burago, M. Gromov, and G. Perelman, A. D. Aleksandrov spaces with curvatures bounded below, Uspekhi Mat. Nauk 47 (1992), no. 2(284), 3-51, 222 (Russian).

[5] F. Klein, Vorlesungen Über die Entwicklung die Mathematik im 19. Jahrhundert, Springer, Berlin, 1926.

[6] K. Menger, Untersuchen über Allgemeine Metrik, Mathematische Annalen 100 (1928), 75113.

[7] Yu. A. Rylov, Description of Riemannian space by means of finite interval, Izvestiya Vysshich Uchebnych Zavedeniyi ser. fis. mat. 132 (1962), no. 3(28), 131-142 (Russian).

[8] _ Relative gravitational field and conservation laws in general relativity, Ann. Physik (7) 12 (1963/1964), 329-353.

[9] __ Extremal properties of Synge's world function and discrete geometry, J. Math. Phys. 31 (1990), no. 12, 2876-2890.

[10] _ Non-Riemannian model of the space-time responsible for quantum effects, J. Math. Phys. 32 (1991), no. 8, 2092-2098.

[11] _ Distorted Riemannian space and technique of differential geometry, J. Math. Phys. 33 (1992), no. 12, 4220-4224.

[12] _ Spacetime distortion as a reason for quantum stochasticity, Chaos-the Interplay Between Stochastic and Deterministic Behaviour (Karpacz, 1995), Springer, Berlin, 1995, pp. 523-530.

[13] _ Description of metric space as a classification of its finite subspaces, Fundamental'aya i Prikladnaya Matematika 7 (2001), no. 4, 1147-1175. 
[14] J. L. Synge, Relativity: The General Theory, North-Holland Publishing, Amsterdam, 1960.

[15] V. A. Toponogov, Riemann spaces with curvature bounded below, Uspehi Mat. Nauk 14 (1959), no. 1 (85), 87-130 (Russian).

Yuri A. Rylov: Institute for Problems in Mechanics, Russian ACADEMy of SCIENCES, 101-1, VeRNADSKII AVENUE, MosCow, 119526, RUSSIA

E-mail address: rylov@i pmnet.ru

URL: http://rsfq1. physics.sunysb.edu/ rylov/yrylov.htm 


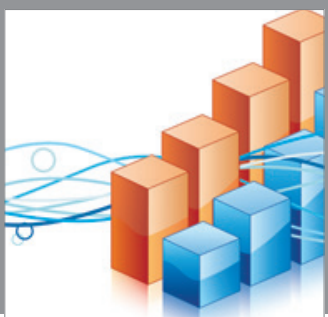

Advances in

Operations Research

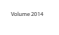

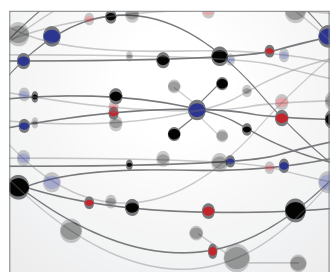

\section{The Scientific} World Journal
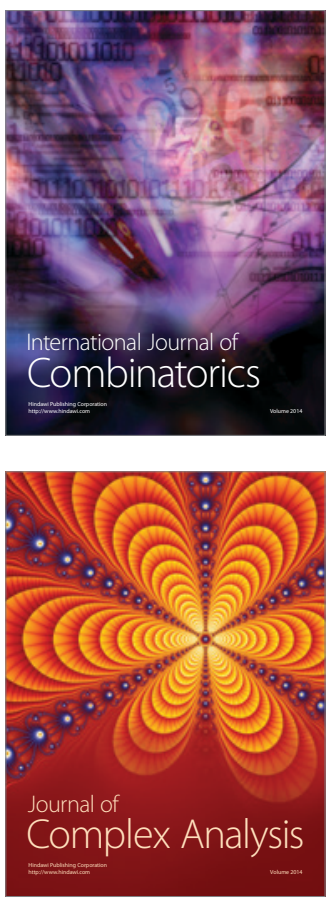

International Journal of

Mathematics and

Mathematical

Sciences
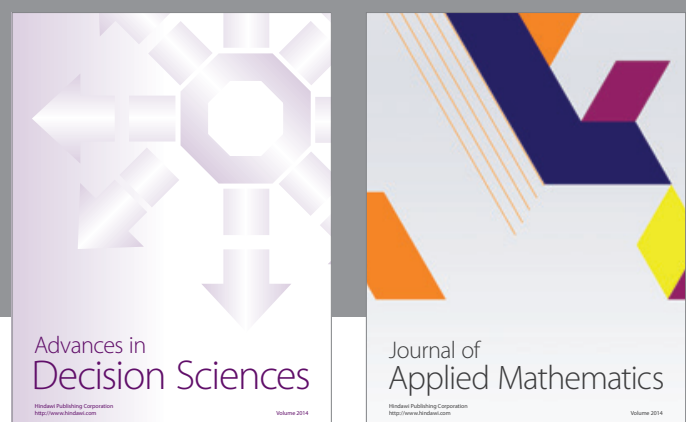

Journal of

Applied Mathematics
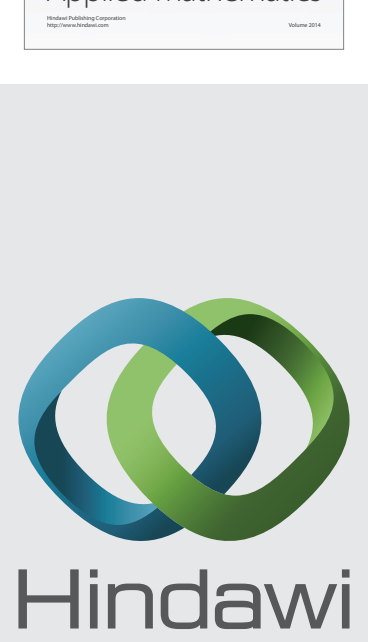

Submit your manuscripts at http://www.hindawi.com
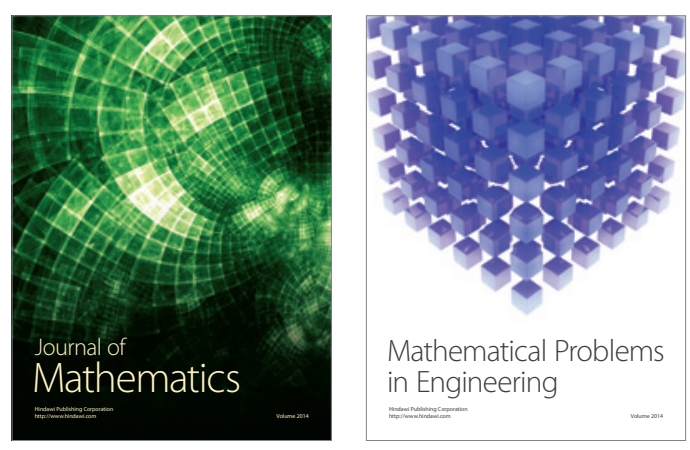

Mathematical Problems in Engineering
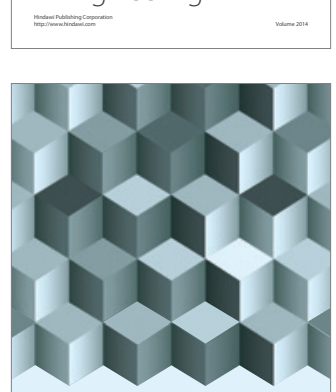

Journal of

Function Spaces
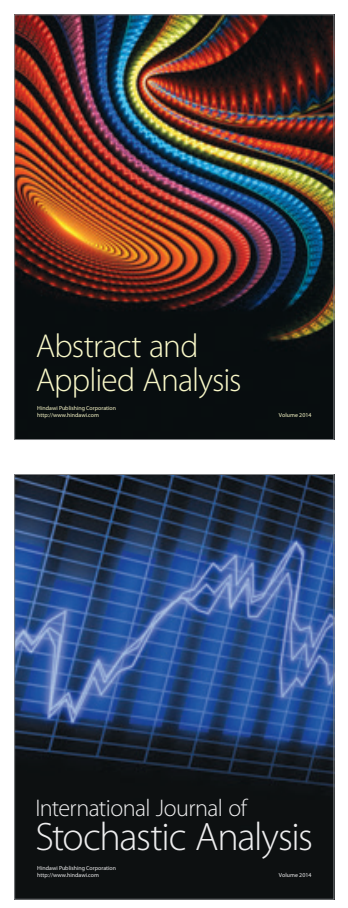

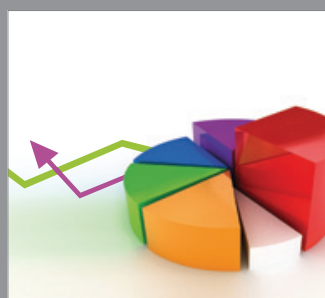

ournal of

Probability and Statistics

Promensencen
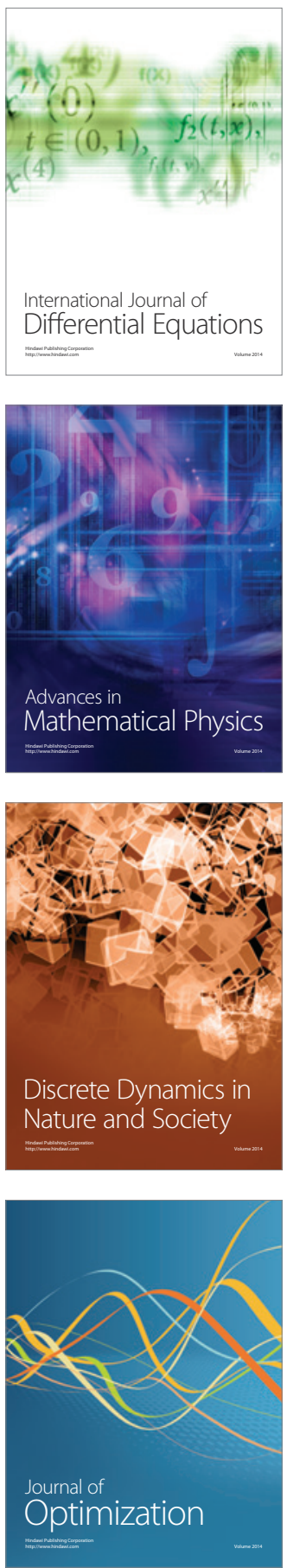